\title{
COMBINATORIAL COVERS AND VANISHING OF COHOMOLOGY
}

\author{
GRAHAM DENHAM ${ }^{1}$, ALEXANDER I. SUCIU², AND SERGEY YUZVINSKY
}

\begin{abstract}
We use a Mayer-Vietoris-like spectral sequence to establish vanishing results for the cohomology of complements of linear and elliptic hyperplane arrangements, as part of a more general framework involving duality and abelian duality properties of spaces and groups. In the process, we consider cohomology of local systems with a general, Cohen-Macaulay-type condition. As a result, we recover known vanishing theorems for rank-1 local systems as well as group ring coefficients, and obtain new generalizations.
\end{abstract}

\section{Contents}

1. Introduction 1

2. Combinatorial covers and spectral sequences 4

3. Submanifold arrangements 10

4. Hyperplane arrangements 14

5. Vanishing of cohomology 21

6. Elliptic arrangements 25

7. Toric complexes and the Cohen-Macaulay property 29

References 32

\section{INTRODUCTION}

1.1. Overview. A remarkable feature of the theory of cohomology jump loci, first brought to light by Eisenbud, Popescu and Yuzvinsky in [EPY03], is that the resonance varieties of hyperplane arrangements "propagate." This raises the natural question: What is the topological underpinning of this phenomenon? Inspired by recent work of Davis, Januszkiewicz, Leary, and Okun [DJLO11], we show that

2010 Mathematics Subject Classification. Primary 55T99. Secondary 14F05, 16E65, 20J05, $32 \mathrm{~S} 22,55 \mathrm{~N} 25$.

Key words and phrases. Combinatorial cover, cohomology with local coefficients, spectral sequence, hyperplane arrangement, elliptic arrangement, toric complex, Cohen-Macaulay property.

${ }^{1}$ Partially supported by NSERC (Canada).

${ }^{2}$ Partially supported by NSF grant DMS-1010298 and NSA grant H98230-13-1-0225. 
complements of hyperplane arrangements, as well as many other spaces have cohomology concentrated in a single degree, for local systems satisfying suitable hypotheses.

Our cohomological vanishing results will be applied in [DSY $]_{1}$ to study the relationship between the duality properties of a space (and its universal abelian cover), and the propagation properties of its characteristic and resonance varieties. We will also apply our machinery in $\left[\mathrm{DSY}_{2}\right]$ to the computation of Novikov homology for arrangement complements, deducing a new bound on the $\Sigma$-invariants for arrangements.

In order to establish such vanishing theorems, we construct here a MayerVietoris-type spectral sequence, which is related to a spectral sequence developed by Davis and Okun in [DO12] in a related context. Our version refines the usual underlying filtration, in order to control the $E_{2}$ page, while requiring weaker hypotheses than those of Davis and Okun. We obtain vanishing results for local systems satisfying Cohen-Macaulay-type conditions on spaces which, in a loose sense, also possess Cohen-Macaulay properties.

1.2. Combinatorial covers and spectral sequences. The input for the spectral sequence is a device which we call a combinatorial cover: that is, a countable cover $\mathcal{C}$ of a space $X$, which is either open, or closed and locally finite, together with an order-preserving, surjective map $\phi: N(\mathcal{C}) \rightarrow P$ from the nerve of the cover to a ranked poset $P$ (with rank function $\rho$ ), for which certain compatibility conditions are satisfied. Given such a cover and a locally constant sheaf $\mathcal{F}$ on $X$, we have a spectral sequence starting at

$$
E_{2}^{p q}=\prod_{x \in P} H^{p-\rho(x)}\left(\phi^{-1}\left(P_{\leq x}\right), \phi^{-1}\left(P_{<x}\right) ; H^{q+\rho(x)}\left(X,\left.\mathcal{F}\right|_{U_{x}}\right)\right),
$$

converging to $H^{p+q}(X, \mathcal{F})$, where $U_{x}=\bigcap_{U \in S} U$, for some set $S \in N(\mathcal{C})$ with $\phi(S)=x$, and $P_{<x}, P_{\leq x}$ are (strict) lower intervals in the poset $P$.

Such covers can be constructed for spaces with a combinatorial stratification: for example, our approach is particularly well-suited when studying an arrangement $\mathcal{A}$ of submanifolds in a smooth manifold $Y$. We assume that each submanifold in $\mathcal{A}$ is either compact or open; moreover, any two submanifolds intersect transversely, and each intersection has only finitely many connected components. We then show that the complement $M(\mathcal{A})$ admits a combinatorial cover indexed by the stratification poset. Moreover, the $E_{2}$ page from (1) specializes to

$$
E_{2}^{p q}=\prod_{X \in P} H^{p-\operatorname{dim}(X)}\left(X, D_{X} ; H^{q+\operatorname{dim}(X)}\left(M(\mathcal{A}),\left.\mathcal{F}\right|_{U_{X}}\right)\right),
$$

where $D_{X}$ is the union of all strata properly contained in $X$.

1.3. Hyperplane arrangements. Now suppose $\mathcal{A}$ is an arrangement of complex hyperplanes in $V=\mathbb{C}^{n}$. We let $L(\mathcal{A})$ be its intersection poset, ordered opposite to inclusion, and $L_{\geq 1}(\mathcal{A})=L(\mathcal{A}) \backslash\{V\}$. For each flat $X \in L_{\geq 1}(\mathcal{A})$, we define an element $\gamma_{X} \in \pi_{1}(M(\mathcal{A}))$ which, upon restriction to the subarrangement $\mathcal{A}_{X}$ 
of hyperplanes containing $X$, lies in the center of the group $\pi_{1}\left(M\left(\mathcal{A}_{X}\right)\right)$. The homology classes $a_{X}=\left[\gamma_{X}\right]$ live in the free abelian group $H_{1}(M(\mathcal{A}), \mathbb{Z})$ and satisfy the compatibility condition $a_{X}=\sum_{H \in \mathcal{A}: H<X} a_{H}$.

We employ the de Concini-Procesi wonderful compactification to construct a particularly convenient combinatorial cover of $M(\mathcal{A})$. Using the spectral sequence (2) associated to this cover, we generalize well-known vanishing results for cohomology with coefficients in rank 1 local systems on arrangement complements, [Ko86, ESV92, STV95].

Previous vanishing results have restricted attention to local systems given by finite-dimensional group representations with field coefficients. In order to understand a vanishing result for (integer) group ring coefficients due to Davis, Januskiewicz, Leary and Okun [DJLO11] within the same framework, however, we note that the restrictions of finite-dimensionality and field coefficients can be avoided.

The classical vanishing result can be formulated as follows: suppose $\mathbb{k}$ is a field and $A$ is a finite-dimensional $\mathbb{k}\left[\pi_{1}(U(\mathcal{A}))\right]$-module where $U(\mathcal{A})$ is the complement of the compactification of $\mathcal{A}$. If the invariant submodule $A^{\gamma_{X}}=0$ for all flats $\left.X \in L_{\geq 1}(\mathcal{A})\right)$ satisfying the Euler characteristic $\chi\left(U\left(\mathcal{A}_{X}\right)\right) \neq 0$, then

$$
H^{p}(U(\mathcal{A}), A)=0 \text { for all } p \neq n-1 .
$$

More generally, we show that $\pi_{1}(U(\mathcal{A}))$ contains free abelian subgroups $C_{S}$ of rank $n$ generated by certain subsets $S$ of the elements $\left\{\gamma_{X}: X \in L_{\geq 1}(\mathcal{A})\right\}$, to wit, the nested sets. We regard $A$ as a module over the (commutative) algebras $\mathbb{k}\left[C_{S}\right]$ by restriction, and formulate a necessary condition for the vanishing result (3) to hold in terms of regular sequences. The classical version is obtained as a special case.

1.4. Elliptic arrangements. Our machinery also applies very well to elliptic arrangements. Let $E$ be an elliptic curve. An elliptic arrangement in $E^{\times n}$ is a finite collection of fibers of group homomorphisms $E^{\times n} \rightarrow E$, see [LV12, Bi13]. We start our analysis by showing that the complement of such an arrangement $\mathcal{A}$ is a Stein manifold, provided $\mathcal{A}$ is essential.

Using our spectral sequence, we obtain a vanishing result analogous to (3). As an application, we recover a recent theorem of Levin and Varchenko [LV12] on vanishing of cohomology for what they term 'convenient' rank 1 local systems. We also infer that the complement of an elliptic arrangement $\mathcal{A}$ is both a duality and an abelian duality space of dimension $n+r$, where $r$ is the corank of $\mathcal{A}$.

A special case of this construction is the configuration space of $n$ points on an elliptic curve $E$, which is a classifying space for the pure elliptic braid group $P E_{n}$. Our approach shows that the group $G=P E_{n}$ is both a duality and an abelian duality group of dimension $n$. Roughly speaking, this means the cohomology of $G$ with coefficients in $\mathbb{Z} G$ and $\mathbb{Z} G^{\text {ab }}$, respectively, is concentrated in degree $n$; for details and further references, we refer to $\left[\mathrm{DSY}_{1}\right]$. 
1.5. Toric complexes. Another context where the techniques developed here apply very well is that of the moment-angle complexes corresponding to the pair $\left(S^{1}, *\right)$. Given a finite, $d$-dimensional simplicial complex $L$ on vertex set $[n]$, the corresponding toric complex, $T_{L}$, is a subcomplex of the $n$-torus, whose $k$-cells are in one-to-one correspondence with the $(k-1)$-simplices of $L$. Notably, the fundamental group $G_{L}=\pi_{1}\left(T_{L}\right)$ is the right-angled Artin group associated to the 1-skeleton of $L$.

A natural condition that comes into play in this setting is that of CohenMacaulayness. The simplicial complex $L$ is said to be a Cohen-Macaulay complex if for each simplex $\sigma \in L$ with the link $\operatorname{lk}(\sigma)$, the reduced cohomology $\widetilde{H}^{*}(\operatorname{lk}(\sigma), \mathbb{Z})$ is concentrated in degree $\operatorname{dim} L-|\sigma|$ and is torsion-free. As shown in [BM01, JM05], the group $G_{L}$, is a duality group if and only if the corresponding flag complex, $\Delta_{L}$, is Cohen-Macaulay.

As a counterpoint, we show that the toric complex $T_{L}$ is an abelian duality space if and only if the simplicial complex $L$ itself is Cohen-Macaulay. Moreover, if $L$ is Cohen-Macaulay over a field $\mathbb{k}$, and $A$ is a maximal Cohen-Macaulay-module over $\mathbb{k}\left[G_{\tau}\right]$, for each $\tau \in L$, we show that the cohomology groups $H^{p}\left(T_{L}, A\right)$ vanish, for all $p \neq d+1$.

\section{Combinatorial covers And SPectral Sequences}

2.1. Combinatorial covers. Let $X$ be a paracompact space, and let $\mathcal{C}$ be a cover of $X$. We can then form the nerve of the cover, $N(\mathcal{C})$ : this is the poset (ordered by inclusion) of all finite collections $S=\left\{U_{1}, \ldots, U_{p}\right\}$ of elements of $\mathcal{C}$ for which $\cap S:=\bigcap_{i=1}^{p} U_{i}$ is non-empty.

Given a poset $P$, we let $|P|$ denote its order complex: this is the simplicial complex with simplices corresponding to chains in $P$. We say $P$ is a ranked poset if it admits a rank function $\rho: P \rightarrow \mathbb{Z}$ compatible with the order and consistent with the covering relation of the order.

Definition 2.1. A combinatorial cover for $X$ is a pair $(\mathcal{C}, \phi)$, where

(1) $\mathcal{C}$ is a countable cover which is either open, or closed and locally finite.

(2) $\phi: N(\mathrm{C}) \rightarrow P$ is an order-preserving, surjective map from the nerve of the cover to a ranked poset $P$, with the property that if $S \leq T$ and $\phi(S)=\phi(T)$, then the inclusion $\cap T \hookrightarrow \cap S$ admits a homotopy inverse.

(3) If $S \leq T$ and $\bigcap S=\bigcap T$, then $\phi(S)=\phi(T)$.

Definition 2.2. We will say that $(\mathcal{C}, \phi)$ is a strong combinatorial cover of $X$ if it is a combinatorial cover and, moreover, the map $\phi$ induces a homotopy equivalence of simplicial complexes, $\phi:|N(\mathcal{C})| \rightarrow|P|$.

Remark 2.3. Any cover $\mathcal{C}$ satisfying condition (1) can be turned into a (strong) combinatorial cover in a trivial fashion: simply take $P=N(\mathcal{C})$ and $\phi$ the identity map of this poset. Typically, however, $P$ is chosen to reflect some underlying combinatorial structure which is independent of the choice of cover. The condition that 


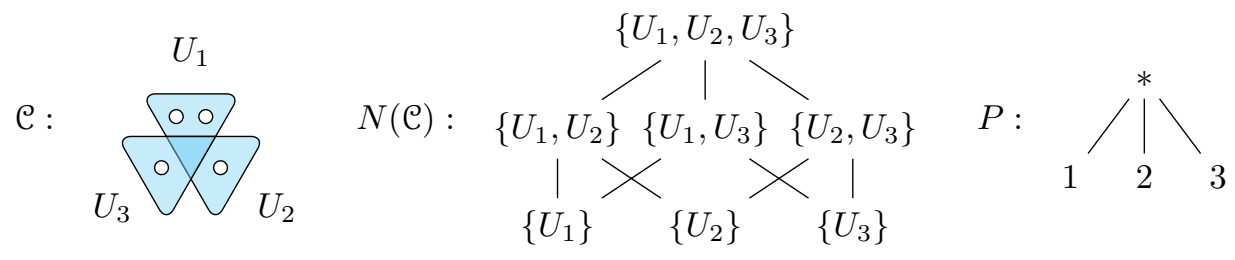

FiguRE 1. A strong combinatorial cover

$\phi$ induces a homotopy equivalence is particularly convenient, but too restrictive for applications like those in $\S 4$.

Example 2.4. Let $X$ be a space homeomorphic to a 2-disk with four punctures, covered by 3 open sets as shown in Figure 1.

Define $\phi: N(\mathcal{C}) \rightarrow P$ by letting $\phi\left(\left\{U_{i}\right\}\right)=i$ for $1 \leq i \leq 3$, and $\phi(S)=*$ if $|S| \neq 1$. To see $(\mathcal{C}, \phi)$ is a combinatorial cover, note that the intersections of any two or more open sets are all identical, so that $\cap S=\cap T$ for any $S, T \in \phi^{-1}(*)$, verifying the only non-vacuous instances of condition (2). In fact, this is a strong combinatorial cover, since the map $\phi:|N(\mathcal{C})| \rightarrow|P|$ is a homotopy equivalence of contractible complexes.

2.2. Sheaf cohomology. Let $\mathbb{k}$ be a (commutative) coefficient ring, $X$ a pathconnected space, and $A$ a left $\mathbb{k}\left[\pi_{1}(X)\right]$-module. We regard $A$ as a local system on $X$. We are interested in the cohomology $H^{\bullet}(X, A)$, and we recall that this is the same as the cohomology $H^{\bullet}(X, \mathcal{F})$ of a locally constant sheaf $\mathcal{F}$ of $\mathbb{k}$-modules determined by $A$. In particular, the stalks $\mathcal{F}_{x} \cong A$ as $\mathbb{k}$-modules for every $x \in X$. Our results apply to locally constant sheaves, then, although (more generally) constructible sheaves appear in some arguments. We note that we do not assume that our stalks are finitely-generated over $\mathbb{k}$, following [Sc03], although the book [Di04] remains a good reference.

Given a space $X$ with an open cover $\mathcal{C}$, there is a tautological map $\pi: X \rightarrow$ $N(\mathcal{C})$, given by $\pi(x)=\left\{i: x \in U_{i}\right\}$. The order topology on $N(\mathcal{C})$ makes $\pi$ continuous. If $\mathcal{F}$ is a locally constant sheaf on $X$, the Leray spectral sequence of $\pi$ unifies Cech cohomology and the Mayer-Vietoris principle:

$$
E_{2}^{p q}=H^{p}\left(N(\mathcal{C}), R^{q} \pi_{*} \mathcal{F}\right) \Rightarrow H^{p+q}(X, \mathcal{F}) .
$$

For $S \in N(\mathcal{C})$, let $[S, \infty)$ denote the principal order ideal, a principal open subset. By definition,

$$
\begin{aligned}
R^{q} \pi_{*} \mathcal{F}([S, \infty)) & =H^{q}\left(U_{S}, i^{*} \mathcal{F}\right) \\
& =H^{q}\left(X,\left.\mathcal{F}\right|_{U_{S}}\right),
\end{aligned}
$$

where $U_{S}=\pi^{-1}([S, \infty))$. Write $\mathcal{H}^{q}=R^{q} \pi_{*} \mathcal{F}$ for short.

For the cohomology of a sheaf in the order topology, we refer to [Deh62]. In particular, since the poset $N(\mathcal{C})$ is the face poset of a simplicial complex, if $\mathcal{G}$ is 
any sheaf, then $H^{\bullet}(N(\mathcal{C}), \mathcal{G})$ can be computed from

$$
C^{p}(N(\mathcal{C}), \mathcal{G}):=\prod_{S \in N_{p}(\mathcal{e})} \mathcal{G}(S)
$$

with a simplicial differential [Deh62, §11.4].

Remark 2.5. Following the notation of [Ive86, IV.8.1], if $Z$ is closed in $X$ and $j: X \backslash Z \hookrightarrow X$ denote the embedding of the complement, then for $p \geq 0$ let

$$
H^{p}(X, Z ; \mathcal{F}):=H^{p}\left(X, j ! j^{*} \mathcal{F}\right) .
$$

In particular, if both $X$ and $X \backslash\{*\}$ are path-connected, and $\mathcal{F}$ is a local system on $X$, then the notation

$$
\widetilde{H}^{p}(X, \mathcal{F}):=H^{p}(X, * ; \mathcal{F})
$$

is well-defined.

Remark 2.6. If $X$ is compact, then we can also interpret (7) as compactly supported cohomology, since then

$$
\begin{aligned}
H^{p}\left(X, j ! j^{*} \mathcal{F}\right) & =H_{c}^{p}\left(X, j ! j^{*} \mathcal{F}\right) \\
& =H_{c}^{p}\left(X \backslash Z, j^{*} \mathcal{F}\right),
\end{aligned}
$$

following [Ive86, III.7.3].

2.3. Poincaré-Verdier duality. For the following classical result, we refer to [Di04, Theorem 3.3.1] in the finite-dimensional case.

Theorem 2.7. Suppose $X$ is a d-dimensional complex manifold, and $\mathcal{F}$ is a locally constant sheaf of $\mathbb{k}$-modules on $X$. If $\mathbb{k}$ is a field, for $0 \leq p \leq 2 d$, we have

$$
H_{c}^{p}(X, \mathcal{F})^{\vee} \cong H^{2 d-p}\left(X, \mathcal{F}^{\vee}\right),
$$

where $-^{\vee}=\operatorname{Hom}(-, \mathbb{k})$. More generally, suppose $\mathbb{k}$ is a principal ideal domain and the stalks of $\mathcal{F}$ are free $\mathbb{k}$-modules. Then, for all $p$, there is a "universal coefficients" short exact sequence,

$$
0 \longrightarrow \operatorname{Ext}_{\mathbb{k}}^{1}\left(H_{c}^{p+1}(X, \mathcal{F}), \mathbb{k}\right) \longrightarrow H^{2 d-p}\left(X, \mathcal{F}^{\vee}\right) \longrightarrow H_{c}^{p}(X, \mathcal{F})^{\vee} \longrightarrow 0 .
$$

Proof. Let $\tilde{\mathbb{k}}$ denote the constant sheaf on $X$. Since $X$ is canonically oriented, we identify its orientation sheaf with $\tilde{\mathbb{k}}$. Then, in the bounded derived category of sheaves on $X$, Verdier duality [KS90, §3.3.1] simplifies to the isomorphism

$$
R \Gamma(X, R \mathcal{H} \operatorname{om}(\mathcal{F}, \tilde{\mathbb{k}}[2 d])) \cong R \operatorname{Hom}_{\mathbb{k}}\left(R \Gamma_{c}(X, \mathcal{F}), \mathbb{k}\right) .
$$

The left side gives rise to a Grothendieck spectral sequence with

$$
\begin{aligned}
E_{2}^{p q} & =H^{p+2 d}\left(X, \mathcal{E x t}{ }^{q}(\mathcal{F}, \tilde{\mathbb{k}})\right) \\
& = \begin{cases}H^{p+2 d}\left(X, \mathcal{F}^{\vee}\right) & \text { if } q=0 \\
0 & \text { otherwise, }\end{cases}
\end{aligned}
$$


using [Bo84, Lemma, p. 126] to reduce the calculation to stalks, together with our assumption that the stalks are free. So this spectral sequence degenerates at $E_{2}$. Computing hyper-Ext on the right-hand side gives another spectral sequence with

$$
E_{2}^{p q}=\operatorname{Ext}_{\mathbb{k}}^{p}\left(H_{c}^{-q}(X, \mathcal{F}), \mathbb{k}\right)
$$

with the same abutment. Then $E_{2}^{p q}=0$ unless $p=0$ or 1 , and the short exact sequences are obtained by comparing the two spectral sequences: this one gives short exact sequences with $E_{\infty}$ in the middle, and the other one identifies $E_{\infty}=$ $H^{\cdot}\left(X, \mathcal{F}^{\vee}\right)$.

Duality gives the following useful trick, which goes back to [Ko86]. Recall that a complex manifold is said to be a Stein manifold if it can be realized as a closed, complex submanifold of some affine space $\mathbb{C}^{k}$. As is well-known, every Stein manifold of (complex) dimension $n$ has the homotopy type of a $\mathrm{CW}$-complex of dimension $n$.

Corollary 2.8. Suppose that $\mathcal{F}$ is a locally constant sheaf of $\mathbb{k}$-modules on a Stein manifold $X$ of dimension $n$, where $\mathbb{k}$ is a principal ideal domain. If the stalks of $\mathcal{F}$ are free $\mathbb{k}$-modules, then $H_{c}^{p}(X, \mathcal{F})=0$ for $0 \leq p<n$.

Proof. Cohomology with coefficients in local systems is an invariant of homotopy type, so the Stein property implies that $H^{n-p}\left(X, \mathcal{F}^{\vee}\right)=0$ for $0 \leq p<n$. By Theorem 2.7,

$$
\operatorname{Ext}_{\mathbb{k}}^{i}\left(H_{c}^{p}(X, \mathcal{F}), \mathbb{k}\right)=0
$$

for $0 \leq p<n$ and all $i$. It follows that $H_{c}^{p}(X, \mathcal{F})=0$ as well.

2.4. A spectral sequence. Now suppose $\mathcal{F}$ is a locally constant sheaf of $\mathbb{k}$ modules on $X$, where $\mathbb{k}$ is either $\mathbb{Z}$, or a field. Suppose also that $X$ admits a combinatorial cover $(\mathcal{C}, \phi)$. We use these data to construct a spectral sequence converging to $H^{\bullet}(X, \mathcal{F})$.

Put an (arbitrary) total order on the elements of $\mathcal{C}$. Then we obtain an exact (Čech) complex of sheaves, $0 \rightarrow \mathcal{F} \rightarrow \mathcal{C} \cdot$, where for $p \geq 0$,

$$
\mathcal{C}^{p}=\mathcal{C}^{p}(\mathcal{C})=\left.\prod_{U_{1}<\cdots<U_{p+1}} \mathcal{F}\right|_{U_{1} \cap \cdots \cap U_{p+1}} .
$$

Here each set $U_{i}$ belongs to the cover $\mathcal{C}$, and the complex $\mathcal{C} \cdot$ has the Cech differential induced by restriction maps: see, for example, [God58, Th. 5.2.1]. More formally, for a subspace $U \stackrel{i}{\hookrightarrow} X$, by $\left.\mathcal{F}\right|_{U}$ we mean the sheaf $i_{*} i^{*} \mathcal{F}$ on $X$, and we note that (1) since $\mathcal{F}$ is locally constant, the higher direct image functors of $i$ vanish, and (2) we also have $\left.\mathcal{F}\right|_{U}=\bar{i}_{!} \bar{i}^{*} \mathcal{F}$, where $\bar{i}$ denotes the inclusion of the closure of $U$ in $X$.

For each integer $q \geq 0$, our hypotheses imply that the subposet $(\rho \phi)^{-1}(q)$ is a disjoint union of incomparable components indexed by $\rho^{-1}(q)$, and for $S$ within 
a single component, the homotopy type of $\cap S$ is constant. We note that any orderpreserving map $\rho: P \rightarrow \mathbb{Z}$ could be used in place of the rank function, provided that the fibers of $\rho$ are antichains.

For each $x \in P$, let $P_{\leq x}=\{y \in P \mid y \leq x\}$. Then $\phi^{-1}\left(P_{\leq x}\right)$ is a sub-poset of $N(\mathcal{C})$. Choosing a set $S \in N(\mathcal{C})$ with $\phi(S)=x$, write $U_{x}=\cap S$; then $U_{x}$ is well-defined up to homotopy.

Given a combinatorial cover $(\mathcal{C}, \phi)$, consider the restriction of $\mathcal{H}^{q}$ to a fiber $\phi^{-1}(x) \subseteq N(\mathcal{C})$. By condition (2), the restriction is locally constant on the connected components of the fiber. For each $x \in P$, let $j_{x}: \phi^{-1}(x) \hookrightarrow N(\mathcal{C})$ denote the inclusion of the fiber.

Theorem 2.9. If $X$ has a combinatorial cover $(\mathcal{C}, \phi)$, then for every locally constant sheaf $\mathcal{F}$ on $X$ there is a spectral sequence with

$$
E_{2}^{p q}=\prod_{x \in P} H^{p-\rho(x)}\left(\phi^{-1}\left(P_{\leq x}\right), \phi^{-1}\left(P_{<x}\right) ; H^{q+\rho(x)}\left(X,\left.\mathcal{F}\right|_{U_{x}}\right)\right),
$$

converging to $H^{p+q}(X, \mathcal{F})$, where $\rho$ denotes the rank function on $P$.

Proof. For each $p \geq 0$, the sheaf $\mathcal{C}^{p}$ is a product indexed by $N_{p}(\mathcal{C})$. Thus, we may form a Cartan-Eilenberg resolution $\mathcal{I}^{\cdot}$ with $\mathcal{I}^{p} \cdot=\prod_{S \in N_{p}(\mathcal{C})} \mathcal{I}_{S}^{p \cdot}$, so that $\mathcal{I}_{S}^{p \cdot}$ is an injective resolution of $\left.\mathcal{F}\right|_{\cap S}$ for each $S \in N_{p}(\mathcal{C})$.

We filter the global sections $\Gamma\left(\mathcal{I}^{*}\right)$ of the double complex by rows. The spectral sequence obtained in this way collapses to $H^{p}\left(X, H^{0}(\mathcal{C} \cdot)\right)=H^{p}(X, \mathcal{F})$, by the acyclicity of $\mathcal{C}$. We may also filter the double complex by setting, for $p \in \mathbb{Z}$,

$$
F^{p}\left(\operatorname{Tot} \Gamma\left(\mathcal{I}^{*}\right)\right)=\prod_{S \in N_{s}(\mathcal{C}): s+\rho(\phi(S)) \geq p} \Gamma\left(\mathcal{I}_{\dot{S}}\right) .
$$

Then the $E_{0}$ term of the filtration spectral sequence is given by

$$
\begin{aligned}
E_{0}^{p q} & =\operatorname{gr}^{p} \operatorname{Tot} \Gamma\left(\mathcal{I}^{* \cdot}\right)^{p+q}, \\
& =\prod_{\substack{S \in N_{s}(\mathcal{C}): s+t=p+q \\
s+\rho(\phi(S))=p}} \Gamma\left(\mathcal{I}^{s, t}\right) . \\
\text {. } &
\end{aligned}
$$

Hence,

$$
\begin{aligned}
E_{1}^{p q} & =\prod_{\substack{S \in N_{s}(\mathcal{C}): s+t=p+q, s+\rho(\phi(S))=p}} H^{t}\left(X,\left.\mathcal{F}\right|_{\cap S}\right), \\
& =\prod_{S \in N_{p-\rho(\phi(S))}(\mathcal{C})} H^{q+\rho(\phi(S))}\left(X,\left.\mathcal{F}\right|_{\cap S}\right) .
\end{aligned}
$$


For a fixed $x \in P$, let $\left(E_{1}^{p q}\right)_{x}$ denote the factors of (17) indexed by $S$ for which $\phi(S)=x$. By construction,

$$
\begin{aligned}
\left(E_{1}^{p q}\right)_{x} & =\prod_{\substack{S \in \phi^{-1}(x): \\
|S|-1+\rho(x)=p}} H^{q+\rho(x)}\left(X,\left.\mathcal{F}\right|_{U_{x}}\right) \\
& =\prod_{\substack{S \in \phi^{-1}\left(P_{\leq x}\right): \\
|S|-1+\rho(x)=p}}\left(j_{x}\right) ! j_{x}^{*} \mathcal{H}^{q+\rho(x)}
\end{aligned}
$$

The differential $d_{1}: E_{1}^{p q} \rightarrow E_{1}^{p+1, q}$ is induced by the Cech differential in $\mathcal{C}$. Suppose that $S \in N_{p}(\mathcal{C}), T \in N_{p+1}(\mathcal{C})$, and $S \subseteq T$. By hypothesis, if $\phi(S)=\phi(T)$, then the inclusion $\cap T \hookrightarrow \cap S$ is a homotopy equivalence; hence, the restriction $\left.\left.\mathcal{F}\right|_{S} \rightarrow \mathcal{F}\right|_{T}$ induces an isomorphism in cohomology. That is, the cohomology sheaf $\mathcal{H}^{q+\rho(x)}$ is locally constant on the open set $\phi^{-1}(x)$. On the other hand, if $\phi(S) \neq \phi(T)$, then $\rho(\phi(S))<\rho(\phi(T))$, by our assumption that fibers of $\rho$ are antichains. It follows in this case that the restriction is zero on $E_{1}^{p q}$. Now, using (6), we have

$$
\left(E_{1}^{p q}\right)_{x}=C^{q+\rho(x)}\left(N(\mathcal{C}),\left(j_{x}\right) ! j_{x}^{*} \mathcal{H}^{q+\rho(x)}\right),
$$

where the differential on the right agrees with the restriction of $d_{1}$. The claim follows.

Corollary 2.10. Suppose $X$ has a strong combinatorial cover $(\mathcal{C}, \phi)$, and $\mathcal{F}$ is a locally constant sheaf on $X$. There is then a spectral sequence with

$$
E_{2}^{p q}=\prod_{x \in P} \widetilde{H}^{p-\rho(x)-1}\left(\mathrm{lk}_{|P|}(x) ; H^{q+\rho(x)}\left(X,\left.\mathcal{F}\right|_{U_{x}}\right)\right),
$$

converging to $H^{p+q}(X, \mathcal{F})$.

Proof. We consider the map of order complexes $|\phi|:|N(\mathcal{C})| \rightarrow|P|$ induced by $\phi$. For any sheaf $\mathcal{G}$ on $N(\mathcal{C})$, we have $\phi_{*}\left(j_{x}\right) ! j_{x}^{*} \mathcal{G}=\left(i_{x}\right) ! i_{x}^{*} \phi_{*} \mathcal{G}$, where $i_{x}:\left|P_{\leq x}\right| \hookrightarrow|P|$.

Since $\left(j_{x}\right)_{!} \mathcal{G}$ is locally constant on each fiber of $\phi$ and the fibers are, by hypothesis, contractible, the Leray spectral sequence of $\phi$ degenerates, so

$$
\begin{aligned}
H^{p-\rho(x)}\left(N(\mathcal{C}),\left(j_{x}\right) ! j_{x}^{*} \mathcal{H}^{q+\rho(x)}\right) & \cong H^{p-\rho(x)}\left(|P|,\left(i_{x}\right) ! i_{x}^{*} \phi_{*} \mathcal{H}^{q+\rho(x)}\right) \\
& \cong \widetilde{H}^{p-\rho(x)-1}\left(\mathrm{lk}_{|P|}(x) ; H^{q+\rho(x)}\left(X,\left.\mathcal{F}\right|_{U_{x}}\right)\right),
\end{aligned}
$$

where the second isomorphism is obtained by using the long exact sequence of the inclusion $i_{x}$, and rewriting the restriction of the sheaf $\phi_{*} \mathcal{H}^{q+\rho(x)}$ as a local system on $\operatorname{lk}_{P}(x)$.

Remark 2.11. For the sake of the readers' intuition, we explain the role of the rank function $\rho$ in the filtration. One obtains the classical Mayer-Vietoris spectral sequence by filtering the complex $\mathcal{I} \cdot$ by the cardinality $s$ of elements of the nerve. Up to reindexing, the $E_{1}$ page of that spectral sequence is the same as our $E_{1}$ page from (17). However, in general, the classical differential $d_{1}$ depends both on the 


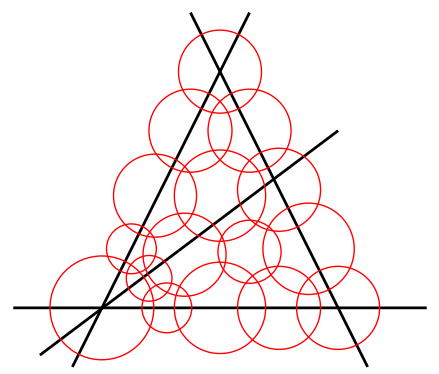

FIgURE 2. A strong combinatorial cover for a line arrangement

combinatorics of the cover as well as the local system, so a closed-form description of the $E_{2}$ page is not available in general.

Davis and Okun solve this problem in [DO12] in a closely related setting by imposing hypotheses ("(Z)" and "(Z')") that are sufficiently restrictive to permit a description of the $E_{2}$ page analogous to that of Theorem 2.9.

Our formalism of combinatorial covers and filtration (15) is chosen to give a spectral sequence with explicit $E_{2}$ page without having to impose hypotheses on the local system. Our filtration is finer than the classical one, in such a way that this $d_{1}$ depends only on the combinatorics of the cover.

\section{Submanifold arrangements}

In [DJLO11], Davis, Januszkiewicz, Leary and Okun use the notion of a strong combinatorial cover (which they call a "small cover") for hyperplane arrangement complements. Their construction restricts an open cover of the ambient affine space to the complement (see Figure 2 for a simple example). In this section, we show that this idea works in greater generality.

3.1. Open covers for submanifold arrangements. To begin, let $Y$ be a smooth, connected manifold, and let $\mathcal{A}=\left\{W_{1}, \ldots, W_{n}\right\}$ be a finite collection of proper, connected submanifolds of $Y$. We will assume that the intersection of any subset of $\mathcal{A}$ is also smooth, and has only finitely many connected components, and call such a collection an arrangement of submanifolds.

Let $L(\mathcal{A})$ denote the collection of all connected components of intersections of zero or more submanifolds. Then $L(\mathcal{A})$ forms a finite poset under inclusion. For every submanifold $X \in L(\mathcal{A})$, let

$$
\mathcal{A}_{X}=\{W \in \mathcal{A}: X \subseteq W\}
$$

be the closed subarrangement associated to $X$, and let

$$
\mathcal{A}^{X}=\left\{W \cap X \mid W \in \mathcal{A} \backslash \mathcal{A}_{X}\right\}
$$

be the restriction of $\mathcal{A}$ to $X$. We say $\mathcal{A}$ is unimodular if each submanifold $X \in L(\mathcal{A})$ is connected. 
Recall that an open cover of a $k$-dimensional manifold is "good" if all sets in the cover and all their nonempty, finite intersections are diffeomorphic to $\mathbb{R}^{k}$. Such covers always exist, and are cofinal in the set of all covers, cf. [BT82, Corollary 5.2]. Compact manifolds, as well as open manifolds (i.e., interiors of compact manifolds with boundary) admit finite good covers.

Now fix a Riemannian metric on $Y$, and let $d$ be the associated distance function. For any subset $X \subset Y$ and $\epsilon>0$, let

$$
X_{\epsilon}=\{y \in Y: d(x, y)<\epsilon \text { for some } x \in X\} .
$$

Theorem 3.1. Let $\mathcal{A}$ be an arrangement of submanifolds in $Y$. There exists a finite open cover $\mathcal{U}$ of $Y$ for which

(1) $\mathcal{U}$ is closed under intersections, and

(2) the poset

$$
L_{U}:=\{X \in L(\mathcal{A}): X \cap \bar{U} \neq \emptyset\}
$$

has a unique minimum, for each $U \in \mathcal{U}$.

If, additionally, each intersection of submanifolds in $\mathcal{A}$ has a finite good cover, then $Y$ has a finite, good open cover $\mathcal{U}$ with the properties above.

Proof. We construct such a cover by induction as follows. Suppose there exist a pair $(L, \mathcal{U})$ where $L$ is an order ideal of $L(\mathcal{A})$, and $\mathcal{U}$ is a finite collection of open sets in $Y$ that covers each element $X \in L$, and which satisfies conditions (1) and (2), above. If $L \neq L(\mathcal{A})$, we show that there exists another pair $\left(L^{\prime}, \mathcal{U}^{\prime}\right)$ with the same property, for which $L^{\prime}$ strictly contains $L$. Since the intersection poset is finite, this implies the claim.

In the notation above, suppose $(L, \mathcal{U})$ is such a pair, and that $X$ is a minimal element of $L(\mathcal{A}) \backslash L$. We let $L^{\prime}=L \cup\{X\}$ and show we can extend the covering $\mathcal{U}$ to $X$. By hypothesis, the function $m: \mathcal{U} \rightarrow L(\mathcal{A})$ defined by $m(U)=\min L_{U}$ is well-defined.

We let

$$
\begin{aligned}
X^{\circ} & =X \backslash \bigcup_{U \in \mathcal{U}} U \\
& =\bigcup_{U \in U} U, X \in L_{U}
\end{aligned}
$$

and claim that there exists a value $\epsilon>0$ for which

(i) For each $U \in \mathcal{U}$, we have $X_{\epsilon}^{\circ} \cap \bar{U} \neq \emptyset \Rightarrow X \cap \bar{U} \neq \emptyset$; and

(ii) For each $X^{\prime} \in L(\mathcal{A})$, we have $X^{\prime} \cap \overline{X_{\epsilon}^{\circ}} \neq \emptyset \Leftrightarrow X \leq X^{\prime}$.

That is, $X_{\epsilon}^{\circ}$ is a neighborhood of $X$, minus the open sets covering the lowerdimensional strata. We claim $X_{\epsilon}^{\circ}$ can be chosen to intersect only (closures of) open sets that already intersect $X$, and to intersect only those strata that contain $X$. 
To argue that such a neighborhood exists, we note that $X$ is closed and $\mathcal{U}$ is finite, so the number

$$
\epsilon_{1}:=\min _{U: X \cap \bar{U}=\emptyset} \inf \{d(x, y): x \in X, y \in \bar{U}\}
$$

is strictly positive. Hence, condition (i) is satisfied whenever $\epsilon<\epsilon_{1}$.

Note that the implication " $\Leftarrow$ " in condition (ii) is satisfied trivially for all $X^{\prime}$ and $\epsilon$. Also note that $X^{\prime} \cap X^{\circ} \neq \emptyset$ implies $X^{\prime} \geq X$. Thus, for the other implication we need to find $\epsilon>0$ such that, for all $X^{\prime} \in L(\mathcal{A})$, we have $X^{\prime} \cap \overline{X_{\epsilon}^{\circ}} \neq \emptyset \Rightarrow$ $X^{\prime} \cap X^{\circ} \neq \emptyset$.

Since each $X^{\prime}$ is closed and $L(\mathcal{A})$ is finite, the number

$$
\epsilon_{2}:=\min _{X^{\prime}: X^{\prime} \cap X^{\circ}=\emptyset} \inf \left\{d(x, y): x \in X^{\prime}, y \in X^{\circ}\right\}
$$

is strictly positive. Therefore, condition (ii) is satisfied if $\epsilon<\epsilon_{2}$.

Let $V=X_{\epsilon}^{\circ}$, for brevity, and put

$$
\mathcal{U}^{\prime}=\mathcal{U} \cup\{V\} \cup\{V \cap U: U \in \mathcal{U} \text { and } V \cap U \neq \emptyset\} .
$$

Clearly, $\mathcal{U}^{\prime}$ is again a finite open cover of $X$. By induction, $\mathcal{U}^{\prime}$ is closed under intersections, Property (1) of the cover. It remains to check Property (2), that the posets $L_{V}$ and $L_{V \cap U}$ have unique minimal elements.

In the former case, condition (ii) directly implies $X$ is the minimal element in $L_{V}$. In the latter case, if $X^{\prime} \cap V \cap U \neq \emptyset$, then $X \leq X^{\prime}$ by (ii). On the other hand, by (i), we have $X \in L_{V \cap U}$, so again $X$ is the (unique) minimal element.

Finally, we assume that each stratum $X$ admits a finite good cover, in which case $X^{\circ}$ also has a finite good cover. Then $X_{\epsilon}^{\circ}$ can be replaced by a finite union of open sets $\{U\}$ for which each such $U$ is diffeomorphic to some $\mathbb{R}^{k}$ and satisfies $m(U)=X$, and similarly with all the intersections.

3.2. A combinatorial cover over the intersection poset. As before, let $\mathcal{A}$ be an arrangement of submanifolds in a manifold $Y$. We now restrict the open cover from Theorem 3.1 to the complement of the arrangement,

$$
M(\mathcal{A})=Y \backslash \bigcup_{W \in \mathcal{A}} W
$$

to obtain a combinatorial cover.

Theorem 3.2. Let $\mathcal{A}$ be an arrangement of submanifolds in a manifold $Y$, and let $M$ be its complement.

(1) If each submanifold $W \in \mathcal{A}$ is either compact or open, then $M$ has a combinatorial cover $(\mathcal{C}, \phi)$ over the intersection poset $L(\mathcal{A})$.

(2) If, moreover, each subspace $X \in L(\mathcal{A})$ is contractible, then $(\mathcal{C}, \phi)$ is a strong combinatorial cover.

Proof. Let $\mathcal{U}$ be the finite open cover of $Y$ given by Theorem 3.1, and let

$$
\mathcal{C}=\{U \cap M: U \in \mathcal{U}\},
$$


an open cover of $M$. Define a map $g: \mathcal{C} \rightarrow L(\mathcal{A})$ by $g(U \cap M)=m(U)$. Clearly, $g$ is an order-reversing map.

The unique minimum property (2) of $\mathcal{U}$ implies that a collection of sets $S=$ $\left\{U_{1}, \ldots, U_{k}\right\}$ in $\mathcal{C}$ has a nonempty intersection if and only if $\left\{g\left(U_{1}\right), \ldots, g\left(U_{k}\right)\right\}$ form a chain in some order. Then $g$ induces a poset map $\phi: N(\mathcal{C}) \rightarrow L(\mathcal{A})$ by letting

$$
\phi(S)=\max _{L(\mathcal{A})}\{g(U): U \in S\} .
$$

We may define a map $\rho: L(\mathcal{A}) \rightarrow \mathbb{Z}$ by letting $\rho(X)=\operatorname{dim} X$ : then, according to Definition 2.2, the pair $(\mathcal{C}, \phi)$ is indeed a combinatorial cover of $M$.

Finally, if each subspace $X \in L(\mathcal{A})$ is contractible, then, by Quillen's Lemma, the above map $\phi$ is a homotopy equivalence. Thus, the triple $(\mathcal{C}, \phi)$ is a strong combinatorial cover.

For the rest of this section we will assume that any two submanifolds in our arrangement $\mathcal{A}$ intersect transversely. Under this assumption, every subspace $X$ in the intersection poset of $\mathcal{A}$ is itself a smooth (not necessarily connected) submanifold of $Y$. Write

$$
D_{X}=\bigcup_{Z \in L(\mathcal{A}): Z<X} Z
$$

Corollary 3.3. Let $\mathcal{A}$ be an arrangement of submanifolds of a manifold $Y$, such that each $W \in \mathcal{A}$ is either compact or open, and any two submanifolds intersect transversely. Let $M$ be the complement of the arrangement, let $(\mathrm{e}, \phi)$ be the combinatorial cover from Theorem 3.2, and let $\mathcal{F}$ be a locally constant sheaf on $M$. There is then a spectral sequence with

$$
E_{2}^{p q}=\prod_{X \in L(\mathcal{A})} H^{p-\rho(X)}\left(X, D_{X} ; H^{q+\rho(X)}\left(M, \mathcal{F}_{U_{X}}\right)\right),
$$

converging to $H^{p+q}(M, \mathcal{F})$. Here $U_{X}$ denotes an open set in $\mathcal{C}$ for which $m\left(U_{X}\right)=$ $X$.

Proof. We note that $N(\mathcal{C})=N(\mathcal{U})$. So the fiber $\phi^{-1}\left(L(\mathcal{A})_{\leq X}\right)$ can be identified with $N\left(\mathcal{U}_{X}\right)$, the nerve of the subcover of $\mathcal{U}$ that intersects $X$. Since $\mathcal{U}$ is a good cover, by the Nerve Lemma, $N\left(\mathcal{U}_{X}\right)$ is homotopic to $X$, and we apply the the spectral sequence from Theorem 2.9.

Remark 3.4. If all submanifolds $W \in A$ are compact, then, by Remark 2.6, we can replace in the above formula the cohomology of the pair $\left(X, D_{X}\right)$ by the compactly supported cohomology of the complement, $X \backslash D_{X}$.

3.3. A combinatorial cover of the arrangement. The previous section constructs a combinatorial cover of the complement of an arrangement whose open sets are local complements. Here, let us cover the arrangement itself, so that the elements of the cover are homotopic to the intersections of submanifolds themselves. Let us further assume that intersections of submanifolds consist of finite unions of equidimensional components. 
Given a submanifold arrangement, let $L^{\prime}(\mathcal{A})$ denote the poset of intersections of one or more submanifolds. Unlike $L(\mathcal{A})$, intersection makes $L^{\prime}(\mathcal{A})$ a meetsemilattice; however, the elements of $L^{\prime}(\mathcal{A})$ need not be connected. For $X$ in $L^{\prime}(\mathcal{A})$, let $\rho(X)=\operatorname{dim} X-k$, where $k$ is the largest number of maximal submanifolds in $\mathcal{A}$ containing $X$ : then if $X \subsetneq Y, \rho(X)<\rho(Y)$. Let

$$
\begin{aligned}
\Sigma(\mathcal{A}) & =\bigcup_{W \in \mathcal{A}} W \\
& =\bigcup_{X \in L^{\prime}(\mathcal{A})} X .
\end{aligned}
$$

For each $X \in L^{\prime}(\mathcal{A})$, let $U_{\epsilon}(X)$ be an open $\epsilon$-neighborhood of the manifold $X$ in $Y$. By our local finiteness hypothesis, if $\epsilon>0$ is small enough, then $U_{\epsilon}(X)$ deformation-retracts to $X$, and $U_{\epsilon}(X \cap Y)=U_{\epsilon}(X) \cap U_{\epsilon}(Y)$ whenever $X \cap Y \neq \emptyset$.

Let $\mathcal{C}=\left\{U_{\epsilon}(X): X \in L^{\prime}(\mathcal{A})\right\}$, and define a map $\phi: N(\mathcal{C}) \rightarrow L^{\prime}(\mathcal{A})^{\text {op }}$ as follows: if $S:=\left(U_{\epsilon}\left(X_{1}\right), \ldots, U_{\epsilon}\left(X_{k}\right)\right) \in N(\mathcal{C})$, let $\phi(S)=X_{1} \cap \cdots \cap X_{k} \in L^{\prime}(\mathcal{A})^{\mathrm{op}}$.

Theorem 3.5. Let $\mathcal{A}$ be an arrangement of submanifolds in a manifold $Y$. Then $(\mathcal{C}, \phi)$ is a strong combinatorial cover of $\Sigma(\mathcal{A})$.

Proof. The claim that $(\mathcal{C}, \phi)$ is a combinatorial cover (Definition 2.2) follows by construction. To see that $\phi$ induces a homotopy equivalence of order complexes, for $X \in L^{\prime}(\mathcal{A})^{\text {op }}$, note that $L^{\prime}(\mathcal{A})_{\leq X}^{\mathrm{op}}$ consists of all intersections of submanifolds that contain $X$. Then $U_{\epsilon}(X)$ is a cone point in the subcomplex $\phi^{-1}\left(L^{\prime}(\mathcal{A})_{\leq X}^{\mathrm{op}}\right)$, so the result follows by Quillen's Lemma [Qu78].

This theorem leads to a spectral sequence complementary to the one of Corollary 3.3:

Corollary 3.6. Let $\mathcal{A}$ be an arrangement of submanifolds in a manifold $Y$, and let $(\mathcal{C}, \phi)$ be the combinatorial cover from Theorem 3.5. For any locally constant sheaf $\mathcal{F}$ on $\Sigma(\mathcal{A})$, there is a spectral sequence with

$$
E_{2}^{p q}=\prod_{X \in L^{\prime}(\mathcal{A})^{\mathrm{op}}} \tilde{H}^{p+\rho(X)-1}\left(\mathrm{l}_{L^{\prime}(\mathcal{A})^{\mathrm{op}}}(X) ; H^{q-\rho(X)}\left(X, \mathcal{F}_{X}\right)\right.
$$

converging to $H^{p+q}(\Sigma(\mathcal{A}), \mathcal{F})$.

Proof. We note that $L^{\prime}(\mathcal{A})^{\text {op }}$ is ranked by $-\rho$, and apply Corollary 2.10.

\section{Hyperplane arRangements}

4.1. Intersection lattice and complement. Let $\mathcal{A}$ be an arrangement of hyperplanes in a finite-dimensional complex vector space $V=\mathbb{C}^{n}$. We will assume throughout that $\mathcal{A}$ is central, that is, all the hyperplanes pass through the origin $0 \in \mathbb{C}^{n}$.

The intersection lattice, $L(\mathcal{A})$, is the ranked poset of all intersections of hyperplanes in $\mathcal{A}$, ordered by reverse inclusion, and ranked by codimension. This is a geometric lattice, with join given by taking the intersection of two flats, and meet 
given by taking the linear span of their sum. For $0 \leq p \leq n$, let $L_{p}(\mathcal{A})$ denote the set of elements of $L(\mathcal{A})$ of rank $p$.

The complement of the arrangement, $M(\mathcal{A})=\mathbb{C}^{n} \backslash \bigcup_{H \in \mathcal{A}} H$, is a connected, smooth complex quasi-projective variety. Moreover, $M(\mathcal{A})$ is a Stein manifold, and thus it has the homotopy type of a CW-complex of dimension at most $n$. In fact, $M(\mathcal{A})$ splits off the linear subspace $\mathbb{C}^{d}=\bigcap_{H \in \mathcal{A}} H$, and so it is homotopic to a cell complex of dimension $n-d$.

The arrangement $\mathcal{A}$ defines by projectivization an arrangement $\overline{\mathcal{A}}$ of codimension 1 projective subspaces in $\mathbb{P}^{n-1}$, with complement $U(\mathcal{A})=\mathbb{P}^{n-1} \backslash \bigcup_{H \in \mathcal{A}} \bar{H}$. The Hopf fibration, $\pi: \mathbb{C}^{n} \backslash\{0\} \rightarrow \mathbb{P}^{n-1}$, restricts to a smooth, principal fibration, $\pi: M(\mathcal{A}) \rightarrow U(\mathcal{A})$, with fiber $\mathbb{C}^{*}$. Fixing a hyperplane $H_{0} \in \mathcal{A}$ yields a trivialization of the bundle map $\mathbb{C}^{n} \backslash H_{0} \rightarrow \mathbb{P}^{n-1} \backslash \bar{H}_{0}$, and thus produces a diffeomorphism $M(\mathcal{A}) \cong \mathbb{C}^{*} \times U(\mathcal{A})$.

Let us fix an ordering of the hyperplanes, $\mathcal{A}=\left\{H_{1}, \ldots, H_{r}\right\}$, and choose linear forms $f_{i}: \mathbb{C}^{n} \rightarrow \mathbb{C}$ with $\operatorname{ker}\left(f_{i}\right)=H_{i}$. Assembling these forms together, we obtain a linear map $f: V \rightarrow \mathbb{C}^{r}$, sending $z \mapsto\left(f_{1}(z), \ldots, f_{r}(z)\right)$. Without much loss of generality, we will assume from now on that $\mathcal{A}$ is essential, i.e., the intersection of all the hyperplanes in $\mathcal{A}$ is precisely 0 , or, equivalently, the map $f$ is injective.

Clearly, $f$ restricts to an inclusion $f: M(\mathcal{A}) \hookrightarrow\left(\mathbb{C}^{*}\right)^{r}$, equivariant with respect to the diagonal action of $\mathbb{C}^{*}$ on both source and target. Thus, $f$ descends to a $\operatorname{map} \bar{f}: M(\mathcal{A}) / \mathbb{C}^{*} \hookrightarrow\left(\mathbb{C}^{*}\right)^{r} / \mathbb{C}^{*}$, which defines an embedding $\bar{f}: U(\mathcal{A}) \hookrightarrow\left(\mathbb{C}^{*}\right)^{r-1}$.

4.2. Flats and meridians. Fix a basepoint $x_{V} \in M(\mathcal{A})$ sufficiently close to 0 . We proceed to describe certain generating sets for the fundamental group of the complement, $G=\pi_{1}\left(M(\mathcal{A}), x_{V}\right)$, and for the "local" fundamental groups, $G_{X}=$ $\pi_{1}\left(M\left(\mathcal{A}_{X}\right), x_{V}\right)$, where recall $\mathcal{A}_{X}=\{H \in \mathcal{A}: H \leq X\}$, for each flat $X \in L(\mathcal{A})$.

Lemma 4.1. Let $X$ be a flat in $L(\mathcal{A})$, and let $i_{X}: M(\mathcal{A}) \rightarrow M\left(\mathcal{A}_{X}\right)$ be the natural inclusion. There is then a basepoint-preserving map $r_{X}: M\left(\mathcal{A}_{X}\right) \rightarrow M(\mathcal{A})$ so that $i_{X} \circ r_{X} \simeq$ id $\left(\right.$ rel $\left.x_{V}\right)$.

Proof. Choose a point $x_{X}$ in the relative interior of $X$ in such a way that the real interval $\left[x_{V}, x_{X}\right]$ intersects only hyperplanes of $\mathcal{A}_{X}$. Let $B_{X}$ be the Minkowski sum of $\left[x_{V}, x_{X}\right]$ with a closed ball of radius $\epsilon$, chosen to be small enough so that again $B_{X} \cap H \neq \emptyset$ if and only if $H \leq X$.

Let $q_{X}: M\left(\mathcal{A}_{X}\right) \rightarrow B_{X} \cap M(\mathcal{A})$ denote the map which is the identity on $B_{X} \cap M\left(\mathcal{A}_{X}\right)$ and radial projection from $x$ to $\partial B_{X}$ on $M\left(\mathcal{A}_{X}\right) \backslash B_{X}$. This is easily seen to be a strong deformation retraction that, moreover, preserves the basepoint (see Figure 3). Composing now the map $q_{X}$ with the inclusion $B_{X} \cap M(\mathcal{A}) \hookrightarrow M(\mathcal{A})$ yields the desired map $r_{X}$.

Let $j: \mathbb{C}^{*} \rightarrow M(\mathcal{A})$ be the inclusion of the fiber of the Hopf fibration containing the basepoint $x_{V}$, and let $j_{\sharp}: \pi_{1}\left(\mathbb{C}^{*}, 1\right) \rightarrow \pi_{1}\left(M(A), x_{V}\right)$ be the induced homomorphism. Upon identifying $\pi_{1}\left(\mathbb{C}^{*}\right)=\mathbb{Z}$, we may define an element $\gamma_{0} \in G$ by

$$
\gamma_{0}=j_{\sharp}(1) .
$$


By construction, $\gamma_{0}$ belongs to the center of $G$.

Similarly, for each flat $X \in L(\mathcal{A})$, we have a central element $\gamma_{0}^{X} \in G_{X}$. Define then an element $\gamma_{X} \in G$ by

$$
\gamma_{X}=\left(r_{X}\right)_{\sharp}\left(\gamma_{0}^{X}\right) .
$$

In particular, for each hyperplane $H \in \mathcal{A}$, we have a "meridian" loop $\gamma_{H}$, based at $x_{V}$. A standard application of the van Kampen theorem shows that the set $\left\{\gamma_{H} \mid H \in \mathcal{A}\right\}$ generates the fundamental group $G=\pi_{1}\left(M(\mathcal{A}), x_{V}\right)$. We also let $a_{X}=\left[\gamma_{X}\right]$ be the corresponding homology classes in $G^{\mathrm{ab}}=H_{1}(M(\mathcal{A}), \mathbb{Z})$.

As noted in [DS14], the homomorphism induced by the map $f: M(\mathcal{A}) \rightarrow$ $\left(\mathbb{C}^{*}\right)^{r}$ on fundamental groups may be identified with the abelianization map, ab: $G \rightarrow G^{\text {ab }}$. Furthermore, the group $G^{\text {ab }}$ is the free abelian group on $\left\{a_{H}: H \in \mathcal{A}\right\}$.

Finally, let $\bar{x}_{V} \in U(\mathcal{A})$ be the image of the basepoint $x_{V} \in M(\mathcal{A})$ under the Hopf map $\pi: M(\mathcal{A}) \rightarrow U(\mathcal{A})$, and, for each flat $X$, let $\bar{\gamma}_{X}=\pi \circ \gamma_{X}$ be the corresponding loop. The fundamental group $\bar{G}=\pi_{1}\left(U(\mathcal{A}), \bar{x}_{V}\right)$ is the quotient of $G$ by the cyclic subgroup $\mathbb{Z}=\left\langle\gamma_{0}\right\rangle$. Moreover, the elements $\left\{\bar{\gamma}_{H} \mid H \in \mathcal{A}\right\}$ generate $\bar{G}$, while the homology classes $\bar{a}_{H}=\left[\bar{\gamma}_{H}\right]$ freely generate the group $\bar{G}^{\text {ab }}=$ $H_{1}(U(\mathcal{A}), \mathbb{Z})$, subject to the single relation $\sum_{H \in \mathcal{A}} \bar{a}_{H}=0$.

4.3. Compatibility of the retraction maps. For each pair of flats $X \leq Y$ in $L(\mathcal{A})$, let $i_{X, Y}: M\left(\mathcal{A}_{Y}\right) \rightarrow M\left(\mathcal{A}_{X}\right)$ denote the inclusion. Recovering our old notation, $i_{X, 0}=i_{X}$. Using the construction of Lemma 4.1, we let

$$
r_{X, Y}=i_{Y} \circ r_{X}: M\left(\mathcal{A}_{X}\right) \rightarrow M\left(\mathcal{A}_{Y}\right) .
$$

Applying Lemma 4.1 to the arrangement $\mathcal{A}_{Y}$, we conclude that $i_{X, Y} \circ r_{X, Y} \simeq$ id, via a basepoint-preserving homotopy. We will need two lemmas.

Lemma 4.2. For any triple of flats $X, Y, Z \in L(\mathcal{A})$, if $X \leq Y$ and $Y \leq Z$, then

(1) $i_{X, Z}=i_{X, Y} \circ i_{Y, Z}$.

(2) $r_{X, Z} \simeq r_{Y, Z} \circ r_{X, Y}$.

In particular, taking $Z=0$, we get $r_{X} \simeq r_{Y} \circ r_{X, Y}$.

Proof. The first claim follows from the definition. To show the second, let $P_{X}$ denote the image of the retract $r_{X}$, for each $X \in L(\mathcal{A})$. In the notation of Lemma 4.1, we have $P_{X}=M\left(\mathcal{A}_{X}\right) \cap B_{X}$. It is enough to show, for any $X \leq Y$, that $r_{Y} \circ i_{Y} \circ r_{X} \simeq r_{X}$. By Lemma 4.1, the corestriction $r_{X}: M\left(\mathcal{A}_{X}\right) \rightarrow P_{X}$ is a homotopy equivalence, so it is enough to show that $r_{Y} \circ i_{Y}: P_{X} \rightarrow M(\mathcal{A})$ is a homotopy equivalence onto its image.

For this, we consider a fiber $\left(r_{Y} \circ i_{Y}\right)^{-1}(x)$ over a point $x$ in the image. If $x \in P_{Y}^{\circ}$, then the fiber consists of just the point $x$. Otherwise, $x$ is in the image of the radial projection towards $x_{Y}$, so the fiber is the intersection of $P_{X}$ with a ray. Since $x \notin Y \subseteq X$, the intersection of the ray with $X$ is the point $x_{Y}$. It follows that the fiber is the intersection of a ray with a convex set (see again Figure 3), hence is contractible. Our claim then follows from the main result of [Sma57]. 


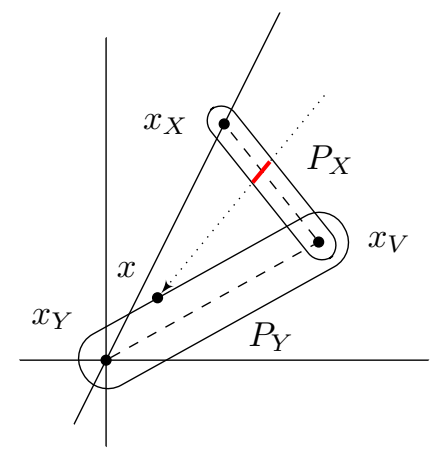

FiguRE 3. The fiber of radial projection

Lemma 4.3. If $H \in \mathcal{A}$ and $X \in L(\mathcal{A})$, and $H \not \leq X$, then the composite $r_{X} \circ i_{X} \circ r_{H}$ is null-homotopic.

Proof. We will continue with the notation above. It is enough to show that image of the restriction $\left.r_{X} \circ i_{X}\right|_{P_{H}}: P_{H} \rightarrow M(\mathcal{A})$ is contractible. Recall that $P_{H}=$ $M\left(\mathcal{A}_{H}\right) \cap B_{H}$, and we claim that $r_{X} \circ i_{X}\left(P_{H}\right)=r_{X} \circ i_{X}\left(B_{H}\right)$.

As in the previous lemma, it is enough to examine a fiber $\left(r_{X} \circ i_{X}\right)^{-1}(x)$ for a point $x \in \partial P_{X}$. Let $R_{x}$ denote the ray through $x_{X}$ and $x$. By hypothesis, $x_{X} \notin H$ and $x \notin H$, so $R_{x} \cap H$ consists of at most one point. Note that $R_{x} \cap\left(B_{H} \backslash P_{H}\right)=$ $R_{x} \cap H$. By slightly varying the radius $\epsilon$ in the definition of $B_{H}$, we may assume that if $\left|R_{x} \cap B_{H}\right|=1$, then $R_{x} \cap B_{H} \cap H=\emptyset$. If $\left|R_{x} \cap B_{H}\right|>1$, then $R_{x} \cap B_{H}=R_{x} \cap P_{H}$, which proves the claim.

By [Sma57] again, the image of $r_{X} \circ i_{X}$ is homotopy equivalent to the contractible set $B_{H}$.

We now consider the restriction of the Hopf fibration to the complement of $\mathcal{A}_{X}$. Let $j_{X}: \mathbb{C}^{*} \rightarrow M\left(\mathcal{A}_{X}\right)$ be the inclusion of the fiber containing the basepoint $x_{V}$. By definition, for $X \leq Y$, we have $j_{X}=i_{X, Y} \circ j_{Y}$. Recalling the definition $\gamma_{0}^{X}=\left(j_{X}\right)_{\sharp}(1)$ for each $X \in L(\mathcal{A})$, we see that for any $X \leq Y$,

$$
\gamma_{0}^{X}=\left(i_{X, Y}\right)_{\sharp}\left(\gamma_{0}^{Y}\right) .
$$

In particular, $\gamma_{0}^{X}=\left(i_{X}\right)_{\sharp}\left(\gamma_{0}\right)$, for all $X$, and by (34), we have

$$
\gamma_{X}=\left(r_{X} \circ i_{X}\right)_{\sharp}\left(\gamma_{0}\right) .
$$

Likewise, in homology, we have $a_{X}=\left(r_{X} \circ i_{X}\right)_{*}\left(\left[\gamma_{0}\right]\right)=\left(r_{X} \circ i_{X} \circ j\right)_{*}(1)$.

For each $X \in L(\mathcal{A})$, let us define an endomorphism of $H_{1}(M(\mathcal{A}), \mathbb{Z})$,

$$
p_{X}=\left(r_{X} \circ i_{X}\right)_{*} .
$$

By Lemma 4.1, we have that $p_{X}^{2}=p_{X}$, for each $X$. 
Proposition 4.4. For all $H \in \mathcal{A}$ and $X \in L(\mathcal{A})$, we have

$$
p_{X}\left(a_{H}\right)= \begin{cases}a_{H} & \text { if } H \leq X ; \\ 0 & \text { otherwise. }\end{cases}
$$

Proof. If $H \leq X$, we write $a_{H}=p_{H}\left(j_{*}(1)\right)$ and calculate:

$$
\begin{aligned}
p_{X}\left(a_{H}\right) & =\left(r_{X} \circ i_{X} \circ r_{H} \circ i_{H} \circ j\right)_{*}(1) \\
& =\left(r_{X, 0} \circ r_{H, X} \circ i_{H} \circ j\right)_{*}(1) \quad \text { by }(35) ; \\
& =\left(r_{H} \circ i_{H} \circ j\right)_{*}(1) \quad \text { by Lemma } 4.2 ; \\
& =a_{H} .
\end{aligned}
$$

If $H \not \subset X$, by Lemma 4.3, we have $\left(r_{X} \circ i_{X} \circ r_{H}\right)_{*}=0$, and the conclusion follows.

The goal of all this was to conclude:

Corollary 4.5. For each flat $X \in L(\mathcal{A})$, the following relation holds

$$
a_{X}=\sum_{H \in \mathcal{A}: H \leq X} a_{H} .
$$

Proof. Using Proposition 4.4 when $X=H$ is a hyperplane, we see that $p_{H}\left(a_{K}\right)=$ $\delta_{H, K} a_{K}$ for $K \in \mathcal{A}$, so $\sum_{H \in \mathcal{A}} p_{H}$ acts as the identity map on $H_{1}(M(\mathcal{A}), \mathbb{Z})$. Thus

$$
\begin{aligned}
a_{X} & =p_{X}\left(\left[\gamma_{0}\right]\right) \\
& =p_{X} \circ \sum_{H \in \mathcal{A}} p_{H}\left(\left[\gamma_{0}\right]\right) \\
& =p_{X}\left(\sum_{H \in \mathcal{A}} a_{H}\right) \\
& =\sum_{H \leq X} a_{H},
\end{aligned}
$$

using Proposition 4.4 again.

4.4. Wonderful compactifications. In their seminal paper [DCP95], De Concini and Procesi constructed natural compactifications of complements of hyperplane arrangements, as follows.

As before, let $\mathcal{A}$ be an arrangement in $\mathbb{C}^{n}$, with complement $M(\mathcal{A})$, and let $\overline{\mathcal{A}}$ be the corresponding arrangement in $\mathbb{P}^{n-1}$, with complement $U(\mathcal{A})$. A building set for the arrangement is a subset

$$
\mathcal{G} \subseteq L_{\geq 1}(\mathcal{A})
$$

which indexes a choice of subspaces that are iteratively blown up in their construction: see [De14] for details, definitions and references. The wonderful compactification, $Y_{\mathcal{G}}$, is a smooth, complete projective variety for which $D:=Y_{\mathcal{G}} \backslash U(\mathcal{A})$ is 
a normal crossings divisor. The components of $D$ are indexed by the building set $\mathcal{G}$ : write

$$
D=\bigcup_{x \in \mathcal{G}} D_{x}
$$

We recall that, for a subset $S \subseteq \mathcal{G}$, the intersection $D_{S}:=\bigcap_{x \in S} D_{x}$ is nonempty if and only if $S$ is a nested set. If $\mathcal{G}=L_{\geq 1}(\mathcal{A})$, we note that nested sets are simply chains in $L(\mathcal{A}) \backslash\{\hat{0}, \hat{1}\}$. We also recall that nested sets form a simplicial complex on the set $\mathcal{G}$ and the maximal element in $L(\mathcal{A})$ is a cone vertex. Let $\mathcal{N}(\mathcal{G})$ denote the link of the cone point, a simplicial complex of dimension $n-2$ with vertex set $\mathcal{G} \backslash\{\hat{1}\}$.

Theorem 4.6. Let $S \in N(\mathcal{G})$ be a nested set. Then the group $\bar{C}_{S}:=\left\langle\bar{\gamma}_{X}: X \in S\right\rangle$ is a free abelian subgroup of $\bar{G}=\pi_{1}(U(\mathcal{A}))$, of rank $k:=|S|$.

Proof. Recall from $\S 4.2$ that $\bar{G}$ is the quotient of $G=\pi_{1}(M(\mathcal{A}))$ by the central subgroup $\mathbb{Z}=\left\langle\gamma_{0}\right\rangle$. Thus, it is enough to prove that the group $C_{S}:=\left\langle\gamma_{X}: X \in\right.$ $S \cup\{0\}\rangle$ is a free abelian subgroup of $G$, of a rank $k+1$. First, we check that if $X, Y \in S$, then $\gamma_{X}$ and $\gamma_{Y}$ commute. If $X \leq Y$, this follows from the discussion above, since $\gamma_{Y}$ is the central element in $G_{Y}$. If $X$ and $Y$ are incomparable, by the nested set property, then there is a linear diffeomorphism

$$
M\left(\mathcal{A}_{X}\right) \times M\left(\mathcal{A}_{Y}\right) \cong M\left(\mathcal{A}_{X \vee Y}\right) .
$$

On the level of fundamental groups, $\gamma_{X}$ and $\gamma_{Y}$ may be identified with central elements in $G_{X}$ and $G_{Y}$, respectively, so they commute as well.

To check that $C_{S}$ is free abelian, we note that the image of $\gamma_{X}$ in $H_{1}(M(\mathcal{A}), \mathbb{Z})=$ $G^{\mathrm{ab}}$ is $a_{X}$, from (39). The elements

$$
\left\{a_{X}: X \in S \cup\{0\}\right\} \subseteq H_{1}(M(\mathcal{A}), \mathbb{Z})
$$

are linearly independent, by [FY04, Prop. 5.2]. Hence, the restriction of the abelianization map gives an epimorphism $C_{S} \rightarrow \mathbb{Z}^{k+1}$. Since $C_{S}$ is abelian and has only $k+1$ generators, this map must be an isomorphism.

Let $U_{S}$ be the intersection of an $\epsilon$-ball around $D_{S}$ in the wonderful compactification $Y_{\mathcal{G}}$ with the hyperplane complement $U(\mathcal{A})$.

Corollary 4.7. The inclusion $i_{S}: U_{S} \hookrightarrow U(\mathcal{A})$ induces an injective map of fundamental groups.

Proof. Note that $U_{S} \simeq\left(\mathbb{C}^{*}\right)^{k}$, a product of $\mathbb{C}^{*}$-orbits passing through $x_{V}$, indexed by the elements $X \in S$. Using formula (37), we see that $\pi_{1}\left(U_{S}, \bar{x}_{V}\right) \cong \mathbb{Z}^{k}$ and the homomorphism $\left(i_{S}\right)_{\sharp}: \pi_{1}\left(U_{S}, \bar{x}_{V}\right) \rightarrow \pi_{1}\left(U(\mathcal{A}), \bar{x}_{V}\right)$ has image the free abelian group $\bar{C}_{S} \cong \mathbb{Z}^{k}$. The conclusion follows from Theorem 4.6. 
4.5. Spectral sequences from the standard embeddings. For a flat $X \in$ $L(\mathcal{A})$, following the standard matroid-theoretic terminology, we say that $X$ is connected if $\beta\left(\mathcal{A}_{X}\right) \neq 0$, where $\beta(\mathcal{A}):=\chi(U(\mathcal{A}))$. This is equivalent to the condition that the closed subarrangement $\mathcal{A}_{X}$ is irreducible (see [Cr67]). In the context of cohomological vanishing results, some authors have also referred to such flats as "dense edges." We note, in particular, that hyperplanes are connected flats.

The construction from [DJLO11] is obtained as follows. If $\mathcal{A}$ is a (central) arrangement in $\mathbb{C}^{n}$, choose a hyperplane $H_{0} \in \mathcal{A}$. Let $Y=\mathbb{C}^{n-1}$, regarded as the affine chart of $\mathbb{P}^{n-1}$ with $H_{0}$ at infinity, and let $W=U(\mathcal{A})$. The indexing poset $L(W, Y)$ can be identified with

$$
P_{H_{0}}:=\left\{X \in L^{\mathrm{op}}(\mathcal{A}): H_{0} \nsucceq X\right\} .
$$

The subsets $X$ are affine spaces, hence contractible. Applying Corollary 3.3, we obtain the following result.

Corollary 4.8. Let $\mathcal{A}$ be a central hyperplane arrangement in $\mathbb{C}^{n}$. For any locally constant sheaf $\mathcal{F}$ on the projectivized complement $U=U(\mathcal{A})$, we have a spectral sequence

$$
E_{2}^{p q}=\prod_{X \in P_{H_{0}}} \widetilde{H}^{p-\rho(X)-1}\left(\left|\left(P_{H_{0}}\right)_{<X}\right| ; H^{q+\rho(X)}\left(U, \mathcal{F}_{U_{X}}\right)\right) \Rightarrow H^{p+q}(U, \mathcal{F}) .
$$

If $\mathcal{A}$ is a (central) arrangement and instead we choose $Y=\mathbb{P}^{n-1}$ and let $U=U(\mathcal{A})$ be the complement of $\overline{\mathcal{A}}$, then the indexing poset of $D=Y \backslash U$ is the truncated intersection poset, $P(\mathcal{A}):=L^{\mathrm{op}}(\mathcal{A}) \backslash\{0\}$. The subsets $\bar{X}$ in the decomposition of $Y$ are projective linear spaces: here, $\rho(X)$ equals the complex dimension of $\bar{X}$, and $\phi^{-1}\left(P(\mathcal{A})_{\leq X}\right)$ is homotopic to the projective space $\bar{X}$. We have a combinatorial cover, and $D_{X} \simeq \phi^{-1}\left(P(\mathcal{A})_{<X}\right)$ is the union of hyperplanes in the (projective) arrangement $\overline{\mathcal{A}^{X}}$, where recall $\mathcal{A}^{X}=\left\{H \cap X \mid H \in \mathcal{A} \backslash \mathcal{A}_{X}\right\}$. For any locally constant sheaf $\mathcal{F}$, our spectral sequence has the form

$$
E_{2}^{p q}=\prod_{X \in P(\mathcal{A})} H_{c}^{p-\rho(X)}\left(U\left(\mathcal{A}^{X}\right) ; H^{q+\rho(X)}\left(U, \mathcal{F}_{U_{X}}\right)\right) \Rightarrow H^{p+q}(U, \mathcal{F}) .
$$

We remark that the cohomology local system in (45) need not be trivial, in contrast with the spectral sequence in [DJLO11, DO12, DS13], where the local system is necessarily trivial.

Example 4.9. If $\mathcal{A}$ consists of 3 distinct lines in $\mathbb{C}^{2}$, then $U=\mathbb{P}^{1} \backslash\{$ three points $\}$. Then

$$
P=\underbrace{}_{0} \begin{array}{lll}
\mathbb{P}^{1} & \\
1 & 2 & 3
\end{array} .
$$

Noting that $F_{2}=\pi_{1}(U, *)$, let $\mathcal{F}$ be the regular representation $\mathbb{k}\left[F_{2}\right]$. The factors of (45) consist of $H_{c}^{p-1}\left(U, \mathbb{k}\left[F_{2}\right]\right)$, indexed by $\mathbb{P}^{1}$, for $q=0$, and three factors $H^{q}\left(S^{1}, \mathbb{k}\left[F_{2}\right]\right)$ indexed by points, for $p=0$. Then $E_{2}^{p q}=E_{3}^{p q}$ have two nonzero 
entries, joined by the differential $d_{3}^{01}$, and the unique nonzero cohomology group $H^{1}\left(U, \mathbb{k}\left[F_{2}\right]\right)=H^{1}\left(F_{2}, \mathbb{k}\left[F_{2}\right]\right)$ is its kernel.

4.6. A spectral sequence from the wonderful compactification. Another combinatorial cover of an arrangement complement can be constructed using the De Concini-Procesi wonderful compactification [DCP95].

Accordingly, Theorem 3.2 provides a combinatorial cover of $U(\mathcal{A})$. This time, the indexing poset is the opposite of the face lattice of $\mathcal{N}(\mathcal{G})$, which we denote $P_{\mathcal{G}}$. For a nested set $S \in \mathcal{N}(\mathcal{G})$, the (complex) dimension of the intersection $D_{S}:=$ $\bigcap_{x \in S} D_{x}$ is $n-1-|S|$ : for ease of notation, we shift the indexing by $n-1$ and let $\rho(S)=-|S|$.

The key advantage of this choice of combinatorial cover is that the open sets $U_{S}$ are complements of $|S|$ smooth components that intersect locally transversally. That is, $U_{S} \simeq\left(S^{1}\right)^{|S|}$, a torus.

The pairs $\left(\phi^{-1}\left(\left(P_{\mathcal{G}}\right)_{\leq S}\right), \phi^{-1}\left(\left(P_{\mathcal{G}}\right)_{<S}\right)\right)$ are pairwise homotopy equivalent to $\left(D_{S}, \bigcup D_{S^{\prime}}\right)$ for faces $S^{\prime} \supsetneq S$ in $\mathcal{N}(\mathcal{G})$. For a nested set $S$ and an element $X \in S$, let us abbreviate

$$
\bigvee S_{<X}:=\bigvee_{Z \in S: Z<X} Z
$$

Using [DCP95, §4.3], one can deduce that

$$
U^{S}:=D_{S} \backslash \bigcup_{S^{\prime} \supsetneq S} D_{S^{\prime}}=\prod_{X \in S} U\left(\mathcal{A}_{X}^{\bigvee S_{<X}}\right) .
$$

The ranks of the arrangements $\mathcal{A}_{X}^{\bigvee S_{<X}}$ add up to $n-1$, again by [DCP95, §4.3], so we see that $U^{S}$ has the homotopy type of an $(n-1-|S|)$-dimensional cell complex.

Applying again Corollary 3.3, we obtain yet another spectral sequence converging to the cohomology of the projectivized complement $U=U(\mathcal{A})$, with coefficients in a locally constant sheaf $\mathcal{F}$. Since each intersection $D_{S}$ is a compact, we will express the $E_{2}$-page in terms of compactly supported cohomology (cf. Remark 3.4).

Corollary 4.10. With notation as above, we have a spectral sequence

$$
E_{2}^{p q}=\prod_{S \in \mathcal{N}(\mathcal{G})} H_{c}^{p+|S|}\left(U^{S} ; H^{q-|S|}\left(\left(S^{1}\right)^{|S|}, \mathcal{F}_{U_{S}}\right)\right) \Rightarrow H^{p+q}(U, \mathcal{F}) .
$$

\section{VANISHING OF COHOMOLOGY}

The main objective of this section is to give conditions for the cohomology of a local system on an arrangement complement to vanish, in terms of certain free abelian subgroups of the fundamental group. These subgroups are fundamental groups of tori embedded in the arrangement complement, and they arise as neighbourhoods around intersections of boundary components in the wonderful compactification of the previous section. 
5.1. Local systems on tori. First, then, we recall the commutative algebra which describes the cohomology of local systems on a torus. Let $\mathbb{k}=\mathbb{Z}$ or a field. If $I$ is an ideal in a commutative, Noetherian $\operatorname{ring} R$ and $A$ is an $R$-module, we say a sequence of elements $g_{1}, \ldots, g_{k} \in$ forms a regular sequence for $A$ if $g_{i}$ is a non-zero divisor on $A /\left(g_{1}, \ldots, g_{i-1}\right) A$, for $1 \leq i \leq k$.

We say that $\operatorname{depth}(I, A)=\infty$ if there exists a regular sequence $g_{1}, \ldots, g_{k}$ for which $A /\left(g_{1}, \ldots, g_{k}\right) A=0$; otherwise, the depth is the length of the longest regular sequence for which $A /\left(g_{1}, \ldots, g_{k}\right) A \neq 0$. If $(R, \mathfrak{m})$ is a local ring, $A$ is a maximal Cohen-Macaulay module when $\operatorname{depth}_{R}(\mathfrak{m}, A)=\operatorname{dim} R$. Depth has a well-known homological interpretation:

Proposition 5.1. For any $R$-module $A$ which is not necessarily finitely-generated, $\operatorname{Ext}_{R}^{p}(R / I, A)=0$ for all $p<\operatorname{depth}_{R}(I, A)$.

This translates directly to a cohomological vanishing condition for local systems on tori.

Lemma 5.2. Suppose $A$ is a local system on $\left(S^{1}\right)^{n}$, for some $n \geq 1$ : that is, a $R:=$ $\mathbb{k}\left[\mathbb{Z}^{n}\right]$-module. Let $\mathbb{1}$ denote the augmentation ideal in $R$. Then $H^{n}\left(\left(S^{1}\right)^{n}, A\right) \cong$ $A / \mathbb{1} A$, and $H^{p}\left(\left(S^{1}\right)^{n}, A\right)=0$ for all $p \leq \operatorname{depth}_{R}(\mathbb{1}, A)$.

Proof. We note that $H^{p}\left(\left(S^{1}\right)^{n}, A\right)=H^{p}\left(\mathbb{Z}^{n}, A\right)=\operatorname{Ext}_{R}^{p}(\mathbb{k}, A)$, and we apply Proposition 5.1.

Remark 5.3. Since the $R$-module $\operatorname{Ext}_{R}^{p}(\mathbb{k}, A)$ is supported at most at the augmentation ideal $\mathbb{1}$, we may localize to note that $H^{p}\left(\left(S^{1}\right)^{n}, A\right)=0$ if and only if $\operatorname{Ext}_{R_{\mathbb{1}}}^{p}\left(\mathbb{k}, A_{\mathbb{1}}\right)=0$. above depends only on the localization $A_{\mathbb{1}}$. That is,

$$
H^{p}\left(\left(S^{1}\right)^{n}, A\right)=0 \Leftrightarrow p \neq n,
$$

if and only if $A_{\mathbb{1}}$ is a maximal Cohen-Macaulay module over $R_{\mathbb{1}}$.

Definition 5.4. With this in mind, we will say that a module $A$ over $R:=\mathbb{k}\left[\mathbb{Z}^{n}\right]$ is a MCM-module if $\operatorname{depth}_{R}(\mathbb{1}, A) \geq n$. Equivalently, the localization $A_{\mathbb{1}}$ is either zero or a maximal Cohen-Macaulay module over $R_{\mathbb{1}}$.

We will frequently consider MCM-modules which arise as follows.

Proposition 5.5. Suppose that $H$ is a free abelian subgroup of a discrete group $G$. Then $\mathbb{k}[G]$ and $\mathbb{k}\left[G^{\mathrm{ab}}\right]$ are both MCM-modules over $\mathbb{k}[H]$.

Proof. The group algebra $\mathbb{k}[G]$ is a free module over $\mathbb{k}[H]$, and a free module over a Cohen-Macaulay ring is maximal Cohen-Macaulay. Since the natural map $H \rightarrow G^{\mathrm{ab}}$ is injective, the same argument applies to $\mathbb{k}\left[G^{\mathrm{ab}}\right]$.

5.2. Cohomology with twisted coefficients. We are now ready to state and prove the main result of this section.

In $\S 4.6$, we constructed a combinatorial cover of $U(\mathcal{A})$ using its embedding in a De Concini-Procesi compactification. This leads to the following vanishing result. 
For an arrangement $\mathcal{A}$, fix a building set $\mathcal{G}$. For each nested set $S \in \mathcal{N}(\mathcal{G})$, let $R_{S}=\mathbb{k}\left[C_{S}\right]$. This is a ring of Laurent polynomials of dimension $|S|$, by Theorem 4.6. We continue to let $\mathbb{1}=\mathbb{1}_{S}$ denote the augmentation ideal in $R_{S}$.

Theorem 5.6. Let $\mathcal{A}$ be a central, essential arrangement of hyperplanes in $\mathbb{C}^{n}$, and $U=U(\mathcal{A})$ its projective complement. Let $A$ be a local system on $U$ over a principal ideal domain $\mathbb{k}$. Suppose that $A$ is a MCM-module over $R_{S}$ for each $S \in \mathcal{N}(\mathcal{G})$. Then $H^{p}(U, A)=0$ for all $p \neq n-1$. If, moreover, $A / \mathbb{1}_{S} A$ is free over $\mathbb{k}$ for each nested set $S$, then so is $H^{n-1}(U, A)$.

Proof. Let $\mathcal{F}$ denote the locally constant sheaf corresponding to $A$. By Corollary 4.10 together with Lemma 5.2, we have a spectral sequence

$$
E_{2}^{p q}=\prod_{S \in \mathcal{N}(\mathcal{G})} H_{c}^{p+|S|}\left(U^{S} ; \operatorname{Ext}_{R_{S}}^{q-|S|}(\mathbb{k}, A)\right) \Rightarrow H^{p+q}(U, \mathcal{F}) .
$$

Since $U^{S}$ is a product of arrangement complements, it is a Stein manifold, and Corollary 2.8 shows compactly supported cohomology of $U^{S}$ in a locally constant sheaf is zero unless $p+|S| \geq n-1-|S|$ : that is, $p \geq n-1-2|S|$.

By Definition 5.4, we have that $\operatorname{depth}_{R_{S}}(\mathbb{1}, A) \geq \operatorname{dim} R_{S}=|S|$ for each ring $R_{S}$ : that is, $\operatorname{Ext}_{R_{S}}^{q-|S|}(\mathbb{k}, A)=0$ for $q-|S| \neq|S|$ : i.e., $q=2|S|$. By Lemma 5.2 again, $\operatorname{Ext}_{R_{S}}^{|S|}(\mathbb{k}, A)=A / \mathbb{1}_{S} A$.

We see that $E_{2}^{p q}=0$ unless $p+q \geq n-1$, so $H^{p}(U, \mathcal{F})=0$ except possibly for $p=n-1$. If, moreover, each entry $E_{2}^{p, n-1-p}$ is free, then so are the submodules $E_{\infty}^{p, n-1-p}$, and $H^{n-1}(U, \mathcal{F})$ as well.

In Proposition 5.5, we saw that $A=\mathbb{k}[G]$ and $A=\mathbb{k}\left[G^{\mathrm{ab}}\right]$ satisfy the hypotheses of the theorem. Other examples can be given in terms of conditions on the group action.

Lemma 5.7. Suppose $A$ is a $\mathbb{k}[G]$-module which either either semisimple or finitedimensional over a field $\mathbb{k}$. If $A^{\gamma_{X}}=0$ for some $X \in \mathcal{G}$, then $A$ is a MCM-module over $R_{S}$ for each nested set $S \in \mathcal{N}(\mathcal{G})$ containing $X$.

Proof. Consider the exact sequence

$$
0 \longrightarrow A^{g} \longrightarrow A \stackrel{1-g}{\longrightarrow} A \longrightarrow A_{g} \longrightarrow 0 .
$$

With either hypothesis, we see $A^{g} \cong 0$ if and only $A_{g} \cong 0$, for any $g \in G$. That is, if $A^{g}=0$, then $1-g$ acts by an automorphism on $A$. By functoriality, $1-g$ induces an automorphism on $\operatorname{Ext}_{R_{S}}(\mathbb{k}, A)$ as well. On the other hand, $1-g$ acts as zero on $\mathbb{k}$, hence on $\operatorname{Ext}_{R_{S}}(\mathbb{k}, A)$. Combining the two, we see $\operatorname{Ext}_{R_{S}}^{p}(\mathbb{k}, A)=0$ for all $p$.

This special case gives a slight generalization of Kohno's classical vanishing result from [Ko86]: 
Corollary 5.8. Let $\mathcal{A}$ be a central, essential complex arrangement, and let $U=$ $U(\mathcal{A})$. Suppose $A$ is a $\mathbb{k}\left[\pi_{1}(U)\right]$-module which is either semisimple or finite-dimensional over a field. If $A^{\gamma_{X}}=0$ for all connected flats $X \in L_{\geq 1}(\mathcal{A})$, then $H^{p}(U, A)=0$ for all $p \neq n-1$.

Proof. We use Theorem 5.6. Let $\mathcal{G}$ be the set of all connected, nonzero flats (the minimal building set, in the language of [DCP95]). Suppose $S \subseteq \mathcal{G}$ is a nonempty nested set and $X \in S$. Using the hypothesis together with Lemma 5.7, we see the only nonzero factor of the spectral sequence (49) is indexed by $S=\emptyset$.

In that case, $W_{\emptyset}=U, R_{\emptyset}=\mathbb{k}$, and $E_{2}^{p q}=0$ unless $q=0$ and $p=n-1$, which implies $H^{p}(U, A)=0$, unless $p=n-1$.

5.3. Weaker vanishing results. Essentially the same arguments gives more information even when the conditions on the $\mathbb{k}\left[\pi_{1}(M(\mathcal{A}))\right]$-module $A$ are relaxed. Here, we briefly consider weaker conditions on $A$.

Let $X$ be a connected space that has the homotopy type of a finite-dimensional $\mathrm{CW}$-complex, and let $n=\operatorname{gd}(X)$ be the minimal dimension of such a CW-complex. If $A$ is a $\pi_{1}(X)$-module, we define

$$
\operatorname{dp}(X ; A)=\max \left\{i: H^{n-i}(X, A) \neq 0\right\},
$$

where by convention $\operatorname{dp}(X ; A)=-1$ if $H^{\bullet}(X, A)=0$. Then Theorem 5.6 admits the following generalization.

Proposition 5.9. Let $A$ be a local system on a projective arrangement complement $U(\mathcal{A})$ of dimension $n-1$. Let $\mathcal{G}$ be a building set for $\mathcal{A}$. Then

$$
\operatorname{dp}(U(\mathcal{A}) ; A) \leq \max _{S \in N(\mathcal{G})}|S|-\operatorname{depth}_{R_{S}}\left(\mathbb{1}_{S}, A\right) .
$$

Proof. Consider the spectral sequence (49) of Theorem 5.6, and a nested set $S$. Once again, the compactly supported cohomology of $U^{S}$ is zero unless $p \geq n-1-$ $2|S|$. By Proposition 5.1, the coefficient module is zero for $q-|S| \geq \operatorname{depth}\left(\mathbb{1}_{S}, A\right)$, so $E_{2}^{p q}=0$ for

$$
p+q<n-1-|S|+\operatorname{depth}\left(\mathbb{1}_{S}, A\right) .
$$

Taking the minimum of the right-hand side over $S \in N(\mathcal{G})$ gives a lower bound on the degree of the first nonzero cohomology group, which translates to the inequality (52).

The following special case includes rank-1 local systems over a field, and uses the same argument as Corollary 5.8.

Corollary 5.10. Suppose a $\mathbb{k}\left[\pi_{1}(U)\right]$-module $A$ is semisimple or finite-dimensional over a field. Then $\operatorname{dp}(U(\mathcal{A}) ; A)$ is at most the largest cardinality of a nested set $S$ having the property that $A^{\gamma_{X}} \neq 0$, for all $X \in S$. 


\section{Elliptic ARRANGEMENTS}

6.1. Abelian arrangements. An abelian arrangement is a finite set of codimension one abelian subvarieties (possibly translated) in a complex abelian variety, see [LV12, Bi13]. Our construction gives a combinatorial cover of the complement of such an arrangement, and thus, a spectral sequence converging to the cohomology of the complement. A special case of the construction is the configuration space of $n$ points on an elliptic curve $E$, which we consider in $\S 6.3$.

The examples we consider are arrangements in a product of complex elliptic curves, $E^{\times n}$. For short, we will refer to these as elliptic arrangements. Let $\mathcal{A}=\left\{H_{1}, \ldots, H_{m}\right\}$ be such an arrangement. The subvarieties $H_{i} \subseteq E^{\times n}$ are fibers of group homomorphisms. Such homomorphisms are parameterized by integer vectors: writing $E$ as an additive group, we may write $H_{i}=f_{i}^{-1}\left(\zeta_{i}\right)$ for some point $\zeta_{i} \in E$, where

$$
f_{i}\left(x_{1}, \ldots, x_{n}\right)=\sum_{j=1}^{n} a_{i j} x_{j},
$$

and $A=\left(a_{i j}\right)$ is a $m \times n$ integer matrix. We let $\operatorname{corank}(\mathcal{A}):=n-\operatorname{rank}(A)$.

The connected components of elements of the intersection lattice of $\mathcal{A}$ are cosets of products of elliptic curves. In particular, the intersection $\Sigma=\bigcap_{H \in \mathcal{A}} H$ is either empty or homeomorphic to a (finite) disjoint union of copies of $E^{\times r}$, where $r$ is the corank of $\mathcal{A}$. We say $\mathcal{A}$ is essential if its corank is zero: clearly, in this case, $m \geq n$.

Proposition 6.1. Let $\mathcal{A}$ be an essential elliptic arrangement. Then the complement $U(\mathcal{A})$ is a Stein manifold.

Proof. Since $\mathcal{A}$ is essential, there exists an $n \times n$ minor of $A$ which is invertible over $\mathbb{Q}$. Without loss, we may assume it is the first $n$ rows, and consider the corresponding subarrangement. Since the complement of an analytic set in a Stein manifold is again Stein, it is sufficient to prove that the complement of the subarrangement is Stein: that is, assume $A$ is a square matrix.

We proceed by induction on the integer value $|\operatorname{det} A|$. If it is one, then $A$ is invertible over $\mathbb{Z}$, in which case let $g: E^{\times n} \rightarrow E^{\times n}$ be the automorphism given by the inverse matrix. Then $\left(f_{i} \circ g\right)\left(x_{1}, \ldots, x_{n}\right)=x_{i}$ for $1 \leq i \leq n$, and so

$$
\begin{aligned}
U(\mathcal{A}) & =E^{\times n} \backslash \bigcup_{1=1}^{n} H_{i} \\
& \cong \prod_{i=1}^{n}\left(E \backslash\left\{\zeta_{i}\right\}\right) .
\end{aligned}
$$

In this case, the claim follows by some classical facts about Stein manifolds (see for instance [FG02]): an open Riemann surface is Stein, and the Stein property is closed under products. 
If $|\operatorname{det} A|>1$, using the Hermite Normal Form via a change of variables as above, we may assume $a_{i j}=0$ for $i>j$ and $a_{i j}<a_{i i}$ for $j<i$. By hypothesis, we have $a_{i i}>1$ for some diagonal entry $1 \leq i \leq n$. Reordering rows and columns, we may take $i=n$.

Consider the arrangement $\mathcal{A}^{\prime}=\left(\mathcal{A} \backslash\left\{H_{n}\right\}\right) \cup\left\{H_{n}^{\prime}\right\}$, where $H_{n}^{\prime}$ is given by the equation $x_{n}+\sum_{j=1}^{n-1} a_{n j} x_{j}=\zeta_{n}$.

Now let $g: E^{\times n} \rightarrow E^{\times n}$ be given by letting $g_{i}=$ id for $1 \leq i<n$ and $g_{n}(x)=a_{n n} x$. The restriction of $g$ to $U(\mathcal{A})$ is a finite, holomorphic map whose image is $U\left(\mathcal{A}^{\prime}\right)$. On the other hand, the elliptic arrangement $\mathcal{A}^{\prime}$ is essential, but the determinant of its coefficient matrix is strictly smaller, so $U\left(\mathcal{A}^{\prime}\right)$ is Stein by induction, and $U(\mathcal{A})$ is Stein by [FG02, Prop. V.1.1].

Corollary 6.2. The complement $U(\mathcal{A})$ of an elliptic arrangement $\mathcal{A}$ in $E^{\times n}$ has the homotopy type of a $C W$-complex of dimension $n+r$, where $r=\operatorname{corank}(\mathcal{A})$.

Proof. If $\mathcal{A}$ is essential, then $U(\mathcal{A})$ is Stein, and thus has the homotopy type of a $n$-dimensional CW-complex. Otherwise, $U(\mathcal{A}) \cong U\left(\mathcal{A}^{\prime}\right) \times E^{\times r}$, where $\mathcal{A}^{\prime}$ is an essential arrangement in $E^{\times(n-r)}$.

The previous section gave a cohomological vanishing result for hyperplane arrangements. The corresponding result for elliptic arrangements can be obtained analogously. Let $\mathcal{A}$ be an elliptic arrangement, with intersection poset $P(\mathcal{A})$. As noted previously, the connected components of intersections of elliptic hyperplanes are cosets of products of elliptic curves. For any $X \in P(\mathcal{A})$, let $T \mathcal{A}_{X}=$ $\{T H\}_{H \in \mathcal{A}: X \subseteq H}$ be the arrangement of tangent hyperplanes to the arrangement at the coset $X \subseteq E^{\times n}$. Now the open set $U_{X}$ for which $m\left(U_{X}\right)=X$ is a strong deformation retract of the (linear) hyperplane arrangement complement $U\left(T \mathcal{A}_{X}\right)$, and the restriction $\mathcal{A}^{X}$ is again an elliptic arrangement.

Theorem 6.3. Let $\mathcal{A}$ be an essential elliptic arrangement of dimension $n$. Suppose that $A$ is a $\mathbb{k}\left[\pi_{1}(U(\mathcal{A}))\right]$-module such that, for each $X \in P(\mathcal{A})$, the module $A$ satisfies the hypotheses of Theorem 5.6 for the space $U\left(T \mathcal{A}_{X}\right)$. Then $H^{i}(U(\mathcal{A}), A)=0$ unless $i=n$. If, moreover, $H^{\bullet}\left(U\left(T \mathcal{A}_{X}\right), A\right)$ is a free $\mathbb{k}$-module for each $X$, then so is $H^{n}(U(\mathcal{A}), A)$.

Proof. We use Corollary 3.3. Let $\mathcal{F}$ denote the locally constant sheaf corresponding to $A$. Since the ambient space is compact, we have a spectral sequence with

$$
E_{2}^{p q}=\prod_{X \in P(\mathcal{A})} H_{c}^{p-\rho(X)}\left(U\left(\mathcal{A}^{X}\right), H^{q+\rho(X)}\left(U\left(T \mathcal{A}_{X}\right), \mathcal{F}_{U_{X}}\right)\right)
$$

which converges to $H^{p+q}(U(\mathcal{A}), \mathcal{F})$. By hypothesis, the coefficient local system is zero unless $q+\rho(X)=n-\rho(X)$, since $U\left(T \mathcal{A}_{X}\right)$ is a central arrangement of rank $n-\rho(X)$.

On the other hand, the complement is a Stein manifold (Proposition 6.1), so by Corollary 2.8, the term indexed by $X$ also vanishes unless $p-\rho(X)=\rho(X)$. Combining, we see that $E_{2}^{p q}=0$ unless $p+q=n$. The last assertion follows as in Theorem 5.6. 
Corollary 6.4. Let $\mathcal{A}$ be an elliptic arrangement in $E^{\times n}$. Then its complement, $U(\mathcal{A})$, is both a duality space and an abelian duality space of dimension $n+r$, where $r$ is the corank of $\mathcal{A}$.

Proof. If $\mathcal{A}$ is essential, then the assertions follow from the previous theorem: if $G=\pi_{1}(U(\mathcal{A}))$ then $\mathbb{k}[G]$ and $\mathbb{k}\left[G^{\mathrm{ab}}\right]$ both satisfy the hypotheses of Theorem 5.6 for each tangent hyperplane arrangement $T \mathcal{A}_{X}$, by Proposition 5.5.

Otherwise, $U(\mathcal{A}) \cong U\left(\mathcal{A}^{\prime}\right) \times E^{\times r}$, where $\mathcal{A}^{\prime}$ is an essential arrangement in $E^{\times(n-r)}$. Since $E^{\times r}$ is both a duality space and an abelian duality space of dimension $2 r$, we are done.

At the other end of the spectrum, we also obtain a vanishing result for rank-1 local systems, comparable to Corollary 5.8:

Corollary 6.5. Let $\mathcal{A}$ be an essential elliptic arrangement, and let $U=U(\mathcal{A})$. Suppose $A$ is a $\mathbb{k}\left[\pi_{1}(U)\right]$-module which is either semisimple or finite-dimensional over a field. Suppose further that $A^{\gamma X}=0$ for all $X \in P(\mathcal{A})$ for which the hyperplane arrangement $T \mathcal{A}_{X}$ is irreducible. Then $H^{p}(U, A)=0$ for all $p \neq n-1$.

6.2. Convenient local systems. Our approach also generalizes a vanishing result of Levin and Varchenko, [LV12, Thm. 5.2]. In the language of forms, these authors consider certain complex, rank-1 local systems on the complement of an elliptic arrangement, which we outline briefly here.

Levin and Varchenko consider local systems on $U=U(\mathcal{A})$ obtained by restricting a rank-1 local system $\mathbb{C}_{\rho}$ on the ambient space, $E^{\times n}$. They impose then a condition on $\mathbb{C}_{\rho}$, which they call convenient, under which it is the case that $H^{p}\left(X,\left.\mathbb{C}_{\rho}\right|_{X}\right)=0$ for all $p$ and all positive-dimensional $X \in P(\mathcal{A})$. They conclude that $H^{p}\left(U,\left.\mathbb{C}_{\rho}\right|_{U}\right)=0$ for all $p<n$, and give a concrete description of $H^{n}\left(U,\left.\mathbb{C}_{\rho}\right|_{U}\right)$. We give the following generalization of their vanishing result from [LV12].

We need a technical result about intervals in the intersection poset $L^{\prime}(\mathcal{A})$, introduced in $\S 3.3$. Let

$$
P^{\prime}(\mathcal{A})=\left\{\{H \in \mathcal{A}: H \supseteq X\} \text { : for some } X \in L^{\prime}(\mathcal{A})\right\},
$$

a poset ordered by reverse inclusion. Clearly $P^{\prime}(\mathcal{A})$ is isomorphic to $L^{\prime}(\mathcal{A})$, by relabelling intersections by the elliptic hyperplanes that contain them. Let $p: P(\mathcal{A}) \rightarrow$ $P^{\prime}(\mathcal{A})$ denote the order-preserving map defined by $p(X)=\{H \in \mathcal{A}: H \supseteq X\}$. If $\mathcal{A}$ is unimodular, then each intersection $X$ is connected, and $p$ is an isomorphism. In general, $p$ is merely surjective.

Lemma 6.6. For any $S \in P^{\prime}(\mathcal{A})$ and $X \in p^{-1}(S)$, the restriction $p:\left(X, E^{\times n}\right) \rightarrow$ $P^{\prime}(\mathcal{A})_{>S}$ induces a homotopy equivalence of order complexes, where $\left(X, E^{\times n}\right)$ denotes an open interval in $P(\mathcal{A})$.

Proof. If $T \subset S$, consider the restriction of the fiber, $p^{-1}\left(P^{\prime}(\mathcal{A})_{\geq T}\right) \cap\left(X, E^{\times n}\right)$. By construction, this set equals

$$
\{Y \in P(\mathcal{A}): X \subseteq Y \text { and } Y \subseteq H \Rightarrow H \in T\} .
$$


The connected component of $\bigcap_{H \in T} H$ containing $X$ is the unique minimal element, so the fiber has a cone point. The result follows by Quillen's Lemma [Qu78].

Theorem 6.7. Let $\mathcal{A}$ be an essential elliptic arrangement in $E^{\times n}$, and $U=U(\mathcal{A})$ the complement. Let $A$ be a local system on $E^{\times n}$ for which $H^{p}\left(X,\left.A\right|_{X}\right)=0$ for all $p$ and positive-dimensional $X \in P(\mathcal{A})$. Then $H^{p}\left(U,\left.A\right|_{U}\right)=0$ for $0 \leq p \leq n-1$.

Proof. Let $\mathcal{F}$ denote the locally constant sheaf with stalk $A$, and $\Sigma(\mathcal{A})=E^{\times n}$ $U(\mathcal{A})$, the union of the elliptic hyperplanes. Let $i, j$ denote the inclusions of $U(\mathcal{A})$ and $\Sigma(\mathcal{A})$, respectively, in $E^{\times n}$. The hypotheses imply $H^{*}\left(E^{\times n}, \mathcal{F}\right)=0$, so the long exact sequence from

$$
0 \longrightarrow i_{i} i^{*} \mathcal{F} \longrightarrow \mathcal{F} \longrightarrow j_{*} j^{*} \mathcal{F} \longrightarrow 0
$$

implies that

$$
H^{p}\left(U(\mathcal{A}), \mathcal{F}_{U(\mathcal{A})}\right) \cong H^{p-1}\left(\Sigma(\mathcal{A}), \mathcal{F}_{\Sigma(\mathcal{A})}\right)
$$

for all $p \geq 0$. By Proposition 6.1, it is enough to show that $H^{p}\left(\Sigma(\mathcal{A}), \mathcal{F}_{\Sigma(\mathcal{A})}\right)=0$ for $p<n-1$.

We cover $\Sigma(\mathcal{A})$ by $\epsilon$-neighborhoods of intersections of elliptic hyperplanes, as in $\S 3.3$, noting that intersections of cosets are cosets, so the elements of $L^{\prime}(\mathcal{A})$ have equidimensional components. We use the spectral sequence from Corollary 3.6, which has

$$
E_{2}^{p q}=\prod_{X \in L^{\prime}(\mathcal{A})^{\mathrm{op}}} \tilde{H}^{p+\rho(X)-1}\left(\operatorname{lk}_{L^{\prime}(\mathcal{A}) \text { op }}(X) ; H^{q-\rho(X)}\left(X, \mathcal{F}_{X}\right)\right),
$$

where $X$ is an intersection of elliptic hyperplanes. By hypothesis, $H^{*}\left(X, \mathcal{F}_{X}\right)=0$ when $X$ is positive-dimensional. If $X$ is zero-dimensional, $\rho(X)=-n$, so $E_{2}^{p q}=0$ unless $q+n=0$. On the other hand, if $X$ is zero-dimensional, the link of $X$ is the realization of the open interval $L^{\prime}(\mathcal{A})_{>X}$, which is homotopic to $\left(X_{0}, E^{\times n}\right)$ for some connected component $X_{0} \in L(\mathcal{A})$, by Lemma 6.6.

On the other hand, $\left[X_{0}, E^{\times n}\right] \cong L\left(T \mathcal{A}_{X_{0}}\right)$, an arrangement of hyperplanes of rank $n$. By Folkman's Theorem [Fo66], then, $\left(X_{0}, E^{\times n}\right)$ has the homotopy type of a wedge of $(n-2)$-spheres, so $\operatorname{lk}_{L^{\prime}(\mathcal{A})^{\text {op }}}(X)$ does as well. It follows that $E_{2}^{p q}=0$ unless $p-n-1=n-2$; i.e., $p+q=n-1$, so $E_{\infty}^{p q}=E_{2}^{p q}$, and the conclusion follows.

6.3. Configuration spaces. Let $M$ be a connected manifold of dimension $m$, and let $F(M, n)$ be the configuration space of $n$ distinct points in $M$, for $n \geq 1$. Let $M_{i j}=\left\{x \in M^{\times n} \mid x_{i}=x_{j}\right\}$. Then

$$
F(M, n)=M^{\times n} \backslash \bigcup_{i<j} M_{i j} .
$$

If $M=\mathbb{C}$, the configuration space $F(\mathbb{C}, n)$ is a complex hyperplane arrangement complement and a classifying space for the pure braid group. 
Now we consider $M=E$, an elliptic curve, in which case $F(E, n)$ is an elliptic arrangement complement. Since $E$ is a topological group, its diagonal action on $E^{\times n}$ gives a principal $E$-bundle,

$$
E \longrightarrow E^{\times n} \stackrel{p}{\longrightarrow} E^{\times n} / E .
$$

Let $\bar{F}(E, n)$ denote the image of $F(E, n)$ under the projection $p$. Since $E$ is abelian, the bundle (61) is trivial: a section $s$ of $p$ is given by lifting a class $[x] \in E^{\times n} / E$ to $x \in E^{\times n}$ with $x_{n}=1$. By restriction,

$$
F(E, n) \cong E \times \bar{F}(E, n),
$$

where $\bar{F}(E, n)$ is an essential elliptic arrangement. Let $P E_{n}=\pi_{1}(F(E, n))$, the pure elliptic braid group (also known as the $n$-string pure braid group on the torus). The configuration space $F(E, n)$ is well-known to be a classifying space for $P E_{n}$, although we note that, for $n \geq 3$, the space $F(E, n)$ is not formal, cf. [Be94, DPS09].

As a special case of Corollary 6.4, we note:

Corollary 6.8. If $A=\mathbb{Z}\left[P E_{n}\right]$ or $A=\mathbb{Z}\left[P E_{n}^{\mathrm{ab}}\right]$, then $H^{p}\left(P E_{n}, A\right)=0$ for $p \neq n+1$, and $H^{n+1}\left(P E_{n}, A\right)$ is a free abelian group. Consequently, for each $n \geq 1$, the pure elliptic braid group $P E_{n}$ is both a duality group and an abelian duality group of dimension $n$.

Remark 6.9. The fact that $P E_{n}$ is a duality group of dimension $n$ can also be deduced from [Ha86, Theorem 4.1]. Indeed, it is shown there that the full elliptic braid group (also known as the mapping class group of the torus with $n$ marked points) is a virtual duality group of dimension $n$. The claim then follows from the fact that $K\left(\pi_{1}\left(P E_{n}, 1\right)\right)=F(E, n)$ is finite-dimensional, and thus $P E_{n}$ is torsion-free.

\section{Toric COMPlexes And the Cohen-Macaulay Property}

7.1. Generalized moment-angle complexes. In this last section, we illustrate our techniques on a class of spaces that arise in toric topology, as a basic example of polyhedral products. For more background and references on the subject, we refer to [BBCG10, Da12, DS07, PS09].

Let $X$ be a connected, finite $\mathrm{CW}$-complex, and let $Y$ a subspace containing a distinguished zero-cell, $*=e^{0}$. Next, let $L$ be a simplicial complex on vertex set $\mathrm{V}$. Associated to these data there is a subcomplex of the cartesian product $X^{\mathrm{V}}$, known as a "generalized moment-angle complex", and defined as

$$
\mathcal{Z}_{L}(X, Y)=\bigcup_{\tau \in L}(X, Y)^{\tau}
$$

where $(X, Y)^{\tau}=\prod_{i \in \mathrm{V}} X_{\tau, i}$, and

$$
X_{\tau, i}= \begin{cases}X & \text { if } i \in \tau ; \\ Y & \text { if not. }\end{cases}
$$


Suppose now that $Y$ is open in $X$, and let $\max (L)$ denote the set of maximal simplices in $L$. By (63), the open sets $\mathcal{C}_{L}:=\left\{(X, Y)^{\tau}: \tau \in \max (L)\right\}$ cover $\mathcal{Z}_{L}(X, Y)$. If $Y \subsetneq X$ are connected manifolds, this cover is a special case of the one constructed in $\S 3.3$.

Lemma 7.1. Regard $\max (L)$ as a (closed) cover of $|L|$. If $Y \subsetneq X$, then $N\left(\mathcal{C}_{L}\right)=$ $N(\max (L))$.

Proof. For any $S \subseteq \max (L)$, it is easy to see that $\bigcap_{\sigma \in S}(X, Y)^{\sigma}=(X, Y)^{\tau}$, where $\tau=\bigcap_{\sigma \in S} \sigma$. With our assumption that $X \neq Y$, we also have $(X, Y)^{\sigma}=(X, Y)^{\tau}$ if and only if $\sigma=\tau$. The claim follows by combining the two assertions.

Here, the intersection poset is $N(\max (L))$, which we can simplify further. Regard $L$ as a poset under reverse inclusion. Let us define an order-preserving $\operatorname{map} \phi: N\left(\mathcal{C}_{L}\right) \rightarrow L$ by

$$
\phi\left((X, Y)^{\tau_{1}}, \ldots,(X, Y)^{\tau_{k}}\right)=\tau_{1} \cap \cdots \cap \tau_{k},
$$

and a rank function $\rho: L \rightarrow \mathbb{Z}$ by $\rho(\sigma)=-|\sigma|$.

Proposition 7.2. If $Y \subsetneq X$, then $\left(\mathcal{C}_{L}, \phi\right)$ is a strong combinatorial cover of $\mathcal{Z}_{L}(X, Y)$.

Proof. It is straightforward to check that $\left(\mathcal{C}_{L}, \phi\right)$ is a combinatorial cover. By Lemma 7.1 and the Nerve Lemma, the map $\phi$ is a homotopy equivalence, which implies it is strong as well.

7.2. Toric complexes. Let $S^{1}=e^{0} \cup e^{1}$ be the circle, endowed with the standard cell decomposition. The resulting moment-angle complex, $T_{L}:=\mathcal{Z}_{L}\left(S^{1}, e^{0}\right)$, is a subcomplex of the $n$-torus $T^{n}$, where $n=|\mathrm{V}|$.

The fundamental group $G_{L}=\pi_{1}\left(T_{L}\right)$ is the right-angled Artin group determined by the graph $\Gamma=L^{(1)}$, with presentation consisting of a generator $v$ for each vertex $v$ in $\mathrm{V}$, and a commutator relation $v w=w v$ for each edge $\{v, w\}$ in $\Gamma$. A classifying space for the group $G_{L}$ is the toric complex $T_{\Delta}$, where $\Delta=\Delta_{L}$ is the flag complex of $L$, i.e., the maximal simplicial complex with 1-skeleton equal to the graph $\Gamma$.

On the other hand, the cohomology $\operatorname{ring} H^{\bullet}\left(T_{L}, \mathbb{k}\right)$ is the exterior StanleyReisner ring $\mathbb{k}\langle L\rangle$, with generators the duals $v^{*}$, and relations the monomials corresponding to the missing faces of $L$.

7.3. Cohen-Macaulay complexes. Recall that an $n$-dimensional simplicial complex $L$ is Cohen-Macaulay if for each simplex $\sigma \in L$, the reduced cohomology $\widetilde{H}^{*}(\operatorname{lk}(\sigma), \mathbb{Z})$ is concentrated in degree $n-|\sigma|$ and is torsion-free. A similar definition holds over a coefficient field $\mathbb{k}$.

For a fixed coefficient ring, the Cohen-Macaulayness of $L$ is a topological property: it depends only on the homeomorphism type of $L$. For $\sigma=\emptyset$, the condition means that $\widetilde{H}^{*}(L, \mathbb{Z})$ is concentrated in degree $n$; it also implies that $L$ is pure, i.e., all its maximal simplices have dimension $n$. 
Remark 7.3. Consider the extreme case in which $L$ is a simplex, so that $T_{L}$ is a torus and $G=\mathbb{Z}^{n}$ for some $n \geq 1$. Then we return to the situation of Lemma 5.2, and we have $H^{p}(G, A)=0$ for $p \neq n$ if and only if either $A$ is a maximal CohenMacaulay module over $\mathbb{k}[G]_{\mathbb{1}}$, or its localization is zero.

Theorem 7.4. Let $L$ be a d-dimensional Cohen-Macaulay complex over $\mathbb{k}$. Suppose $A$ is a MCM-module over $\mathbb{k}\left[G_{\tau}\right]$, for each $\tau \in L$. Then $H^{p}\left(T_{L}, A\right)=0$, for all $p \neq d+1$. If, moreover, $A$ is a free $\mathbb{k}$-module, then so is $H^{d+1}\left(T_{L}, A\right)$.

Proof. Replace $T_{L}$ by a homotopy equivalent complex $\mathcal{Z}_{L}\left(S^{1}, Y\right)$, where $Y$ is a contractible open neighbourhood of $e^{0}$. Then it is enough to show the corresponding vanishing property for $H^{p}\left(\mathcal{Z}_{L}\left(S^{1}, Y\right), \mathcal{F}\right)$, where $\mathcal{F}$ is the locally constant sheaf on $\mathcal{Z}_{L}\left(S^{1}, Y\right)$ defined by $A$.

The space $\mathcal{Z}_{L}\left(S^{1}, Y\right)$ has an open, strong combinatorial cover from Proposition 7.2. The spectral sequence of Corollary 2.10 has $E_{2}$ term

$$
E_{2}^{p q}=\bigoplus_{\tau \in L} H_{c}^{p+|\tau|}\left(\operatorname{st}_{L}(\tau) \backslash \mathrm{k}_{L}(\tau) ; H^{q-|\tau|}\left(\left(S^{1}, Y\right)^{\tau},\left.\mathcal{F}\right|_{\tau}\right)\right) .
$$

Since the complement $\operatorname{st}_{L}(\tau) \backslash \mathrm{k}_{L}(\tau)$ is contractible, the coefficient local system is necessarily trivial. Moreover, $\left(S^{1}, Y\right)^{\tau} \simeq T^{|\tau|}$, a torus, so by Remark 7.3,

$$
H^{q}\left(T^{|\tau|},\left.\mathcal{F}\right|_{\tau}\right)=H^{q}\left(T^{|\tau|}, \operatorname{res}_{G_{\tau}}^{G_{L}} A\right)= \begin{cases}A_{G_{\tau}} & \text { if } q=|\tau| \\ 0 & \text { otherwise. }\end{cases}
$$

It follows that

$$
E_{2}^{p q}=\bigoplus_{\tau \in L: q=2|\tau|} H_{c}^{p+|\tau|}\left(\mathrm{st}_{L}(\tau) \backslash \mathrm{lk}_{L}(\tau) ; A_{G_{\tau}}\right) .
$$

By our assumption that $L$ is a $d$-dimensional Cohen-Macaulay complex over $\mathbb{k}$, we know that

$$
\widetilde{H}^{p+|\tau|-1}\left(\mathrm{lk}_{L}(\tau), A_{G_{\tau}}\right)=0,
$$

except possibly for $p+|\tau|-1=d-|\tau|$. In that case, $E_{2}^{p q}=0$, except possibly for $p+q=d+1$. Thus, the spectral sequence collapses at the $E_{2}$ page, and the claim follows from Corollary 2.10.

If, moreover, $A$ is free over $\mathbb{k}$, so is any module of coinvariants. If $L$ is CohenMacaulay, then the nonzero summands of (68) are free as well, and it follows that $H^{d+1}\left(T_{L}, A\right)$ is too.

In a special case, we obtain a converse, along the lines of [BM01, Theorem C]:

Theorem 7.5. If $\mathbb{k}=\mathbb{Z}$ or a field, and $A=\mathbb{k}\left[G_{L}^{\mathrm{ab}}\right]$, then $L$ is a d-dimensional Cohen-Macaulay complex over $\mathbb{k}$ if and only if $H^{p}\left(T_{L}, A\right)=0$ for all $p \neq d+1$ and $H^{d+1}\left(T_{L}, A\right)$ is a free $\mathbb{k}$-module.

Proof. To check the implication " $\Rightarrow$ ", we note that $A$ is a MCM-module, by Proposition 5.5, and a free $\mathbb{k}$-module. The result follows from Theorem 7.4. 
To check the converse, suppose $L$ has dimension $d$, cohomology vanishes, but $L$ is not Cohen-Macaulay. First suppose $\mathbb{k}$ is a field. Since $A$ is a ring, by naturality of restriction, $H^{*}\left(\left(S^{1}, Y\right)^{\tau},\left.\mathcal{F}\right|_{\tau}\right)$ is an $A$-module for all $\tau$. Then, as above,

$$
\widetilde{H}^{p+|\tau|-1}\left(\mathrm{lk}_{L}(\tau), A_{G_{\tau}}\right) \neq 0
$$

for some $p<d-2|\tau|+1$, so $E_{2}^{p q} \neq 0$ where $q=2|\tau|$. Our hypothesis implies $E_{\infty}^{p q}=0$ unless $p+q=d+1$, so for some (least) integer $r \geq 2$, the differential $d_{r}^{p q}$ is nonzero. By construction, summands of the target of $d_{r}^{p q}$ are indexed by those simplices $\sigma$ for which $\sigma \subsetneq \tau$, so by restricting and projecting, there exists a simplex $\sigma$ and a nonzero map

$$
d(\tau, \sigma): \widetilde{H}^{p+|\tau|-1}\left(\mathrm{lk}_{L}(\tau), A_{G_{\tau}}\right) \rightarrow \widetilde{H}^{p+|\sigma|-1}\left(\mathrm{lk}_{L}(\sigma), A_{G_{\sigma}}\right) .
$$

We note that $A_{G_{\tau}}=A / I_{\tau}$, where $I_{\tau}$ is prime ideal of $A$. so $d$ is a homomorphism of modules over the domain $A_{G_{\sigma}}$. Since $\mathbb{k}$ is a field, $\widetilde{H}^{p+|\sigma|-1}\left(\mathrm{k}_{L}(\sigma), A_{G_{\sigma}}\right)$ is a free module. The ideal $I_{\tau}$ annihilates $(70)$, so it annihilates the image of $d(\tau, \sigma)$. Since $I_{\sigma} \subsetneq I_{\tau}$, the image of $I_{\tau}$ is nonzero in $A_{G_{\sigma}}$. It follows that $d(\tau, \sigma)$ is the zero map, a contradiction.

Now suppose $\mathbb{k}=\mathbb{Z}$. By the Universal Coefficients Theorem and the previous argument, $L$ is Cohen-Macaulay over all fields. Then $L$ is Cohen-Macaulay over $\mathbb{Z}$, again by Universal Coefficients.

\section{REFERENCES}

[BBCG10] Anthony Bahri, Martin Bendersky, Frederick R. Cohen, Samuel Gitler, The polyhedral product functor: a method of computation for moment-angle complexes, arrangements and related spaces, Advances in Math. 225 (2010), no. 3, 1634-1668. MR2673742 (2012b:13053) 7.1

[Be94] Roman Bezrukavnikov, Koszul DG-algebras arising from configuration spaces, Geom. Funct. Anal. 4 (1994), no. 2, 119-135. MR1262702 (95c:55011) 6.3

[Bi13] Christin Bibby, Cohomology of abelian arrangements, arXiv:1310.4866v3. 1.4, 6.1

[Bo84] Armand Borel et al., Intersection cohomology, Progress in Mathematics, vol. 50, Birkhäuser, Boston, 1984. MR0788171 (88d:32024) 2.3

[BT82] Raoul Bott and Loring Tu, Differential forms in algebraic topology, Graduate Texts in Mathematics, vol. 82, Springer-Verlag, New York-Berlin, 1982. MR0658304 (83i:57016) 3.1

[BM01] Noel Brady and John Meier, Connectivity at infinity for right angled Artin groups, Trans. Amer. Math. Soc. 353 (2001), no. 1, 117-132. MR1675166 (2001b:20068) 1.5, 7.3

[DCP95] Corrado De Concini and Claudio Procesi, Wonderful models of subspace arrangements, Selecta Math. (N.S.) 1 (1995), no. 3, 459-494. MR1366622 (97k:14013) 4.4, 4.6, 4.6, 4.6, 5.2

[Cr67] Henry Crapo, A higher invariant for matroids, J. Combinatorial Theory 2 (1967), 406-417. MR0215744 (35 \#6579) 4.5

[Da12] Michael W. Davis, Right-angularity, flag complexes, asphericity, Geom. Dedicata 159 (2012), no. 1, 239-262. MR2944529 7.1

[DJLO11] Michael W. Davis, Ian Leary, Tadeusz Januszkiewicz, and Boris Okun, Cohomology of hyperplane complements with group ring coefficients, Intern. Math. Res. Notices 2011, no. 9, 2110-2116. MR2806559 1.1, 1.3, 3, 4.5, 4.5 
[DO12] Michael W. Davis and Boris Okun, Cohomology computations for Artin groups, Bestvina-Brady groups, and graph products, Groups Geom. Dyn. 6 (2012), no. 3, 485-531. MR2961283 1.1, 2.11, 4.5

[DS13] Michael W. Davis and Simona Settepanella, Vanishing results for the cohomology of complex toric hyperplane complements, Publ. Mat. 57 (2013), no. 2, 379-392. MR3114774 4.5

[Deh62] René Deheuvels, Homologie des ensembles ordonnés et des espaces topologiques, Bull. Soc. Math. France 90 (1962), 261-321. MR0168614 (29 \#5874) 2.2, 2.2

[De14] Graham Denham, Toric and tropical compactifications of hyperplane complements, Ann. Fac. Sci. Toulouse Math. 23 (2014), no. 2, 297-333. MR3205595 4.4

[DS07] Graham Denham and Alexander I. Suciu, Moment-angle complexes, monomial ideals, and Massey products, Pure Appl. Math. Q. 3 (2007), no. 1, 25-60. MR2330154 (2008g:55028) 7.1

[DS14] Graham Denham and Alexander I. Suciu, Multinets, parallel connections, and Milnor fibrations of arrangements, Proc. London Math. Soc. 108 (2014), no. 6, 1435-1470. MR3218315 4.2

[DSY ${ }_{1}$ Graham Denham, Alexander I. Suciu, and Sergey Yuzvinsky, Abelian duality and propagation of resonance, in preparation. 1.1, 1.4

[DSY $\mathrm{DS}_{2} \quad$ Graham Denham, Alexander I. Suciu, and Sergey Yuzvinsky, Vanishing Novikov homology, in preparation. 1.1

[Di04] Alexandru Dimca, Sheaves in topology, Universitext, Springer-Verlag, Berlin, 2004. MR2050072 (2005j:55002) 2.2, 2.3

[DPS09] Alexandru Dimca, Stefan Papadima, and Alexander I. Suciu, Topology and geometry of cohomology jump loci, Duke Math. Journal 148 (2009), no. 3, 405-457. MR2527322 (2011b:14047) 6.3

[EPY03] David Eisenbud, Sorin Popescu, and Sergey Yuzvinsky, Hyperplane arrangement cohomology and monomials in the exterior algebra, Trans. Amer. Math. Soc. 355 (2003), no. 11, 4365-4383. MR1986506 (2004g:52036) 1.1

[ESV92] Hélène Esnault, Vadim Schechtman, and Eckart Viehweg, Cohomology of local systems on the complement of hyperplanes, Invent. Math. 109 (1992), 557-561. MR1176205 (93g:32051) Erratum, ibid. 112 (1993), no. 2, 447. MR1213111 (94b:32061) 1.3

[FY04] Eva Maria Feichtner and Sergey Yuzvinsky, Chow rings of toric varieties defined by atomic lattices, Invent. Math. 155 (2004), no. 3, 515-536. MR2038195 (2004k:14009) 4.4

[Fo66] Jon Folkman, The homology groups of a lattice, J. Math. Mech. 15 (1966), 631-636. MR0188116 (32 \#5557) 6.2

[FG02] Klaus Fritzsche and Hans Grauert, From holomorphic functions to complex manifolds, Grad.Texts in Math., vol. 213. Springer-Verlag, New York, 2002. MR1893803 (2003g:32001) 6.1

[God58] Roger Godement, Topologie algébrique et théorie des faisceaux, Actualités Sci. Ind. no. 1252, Publ. Math. Univ. Strasbourg, no. 13, Hermann, Paris, 1958 MR0102797 (21 \#1583) 2.4

[Ha86] John Harer, The virtual cohomological dimension of the mapping class group of an orientable surface, Invent. Math. 84 (1986), no. 1, 157-176. MR0830043 (87c:32030) 6.9

[Ive86] Birger Iversen, Cohomology of sheaves, Universitext, Springer-Verlag, Berlin, 1986. MR842190 (87m:14013) 2.5, 2.6

[KS90] Masaki Kashiwara and Pierre Schapira, Sheaves on manifolds, Grundlehren Math. Wiss., vol. 292, Springer-Verlag, Berlin, 1990. MR1074006 (92a:58132) 2.3 
[JM05] Craig Jensen and John Meier, The cohomology of right-angled Artin groups with group ring coefficients, Bull. London Math. Soc. 37 (2005), no. 5, 711-718. MR2164833 (2006m:20054) 1.5

[Ko86] Toshitake Kohno, Homology of a local system on the complement of hyperplanes, Proc. Japan Acad. Ser. A Math. Sci. 62 (1986), no. 4, 144-147. MR0846350 (87i:32019) 1.3, 2.3, 5.2

[LV12] Andrey Levin and Alexander Varchenko, Cohomology of the complement to an elliptic arrangement, in: Configuration spaces: geometry, combinatorics and topology, 373388, CRM Series, vol. 14, Ed. Norm., Pisa, 2012. MR3203648 1.4, 6.1, 6.2

[PS09] Stefan Papadima and Alexander I. Suciu, Toric complexes and Artin kernels, Adv. Math. 220 (2009), no. 2, 441-477. MR2466422 (2010h:57007) 7.1

[Qu78] Daniel Quillen, Homotopy properties of the poset of nontrivial p-subgroups of a group, Adv. in Math. 28 (1978), no. 2, 101-128. MR0493916 (80k:20049) 3.3, 6.2

[STV95] Vadim Schechtman, Hiroaki Terao, and Alexander Varchenko, Local systems over complements of hyperplanes and the Kac-Kazhdan condition for singular vectors, J. Pure Appl. Algebra 100 (1995), no. 1-3, 93-102. MR1344845 (96j:32047) 1.3

[Sc03] Jörg Schürmann, Topology of singular spaces and constructible sheaves, Mathematics Institute of the Polish Academy of Sciences, Mathematical Monographs, vol. 63, Birkhäuser Verlag, Basel, 2003. MR2031639 (2005f:32053) 2.2

[Sma57] Stephen Smale, A Vietoris mapping theorem for homotopy, Proc. Amer. Math. Soc. 8 (1957), 604-610. MR0087106 (19,302f) 4.3, 4.3

Department of Mathematics, University of Western Ontario, London, On N6A $5 \mathrm{~B} 7$

E-mail address: gdenham@uwo.ca

URL: http://www.math.uwo.ca/ gdenham

Department of Mathematics, Northeastern University, Boston, MA 02115

E-mail address: a.suciu@neu.edu

$U R L:$ http://www. northeastern.edu/suciu/

Department of Mathematics, University of Oregon, Eugene, OR 97403

E-mail address: yuz@uoregon.edu

$U R L:$ http://pages .uoregon.edu/yuz/ 